\title{
Dark Matter Astrophysics
}

\author{
Guido D’Amico, Marc Kamionkowski and Kris Sigurdson
}

\begin{abstract}
These lectures are intended to provide a brief pedagogical review of dark matter for the newcomer to the subject. We begin with a discussion of the astrophysical evidence for dark matter. The standard weakly-interacting massive particle (WIMP) scenario - the motivation, particle models, and detection techniques-is then reviewed. We provide a brief sampling of some recent variations to the standard WIMP scenario as well as some alternatives (axions and sterile neutrinos). Exercises are provided for the reader. ${ }^{1}$
\end{abstract}

\section{Introduction}

Dark matter is an essential ingredient in a good fraction of the literature on extragalactic astronomy and cosmology. Since dark matter cannot be made of any of the usual standard-model particles (as we will discuss below), dark matter is also a central focus of elementary-particle physics. The purpose of this review is to provide a pedagogical introduction to the principle astrophysical evidence for dark matter and to some of the particle candidates.

Rather than present a comprehensive survey of the vast and increasingly precise measurements of the amount and distribution of dark matter, we will present very

Guido D'Amico

SISSA, via Beirut 2-4, 34014 Trieste, Italy; e-mail: damico@sissa.it

Marc Kamionkowski

California Institute of Technology, Mail Code 350-17, Pasadena, CA 91125, USA;

e-mail:kamion@caltech.edu

Kris Sigurdson

Department of Physics and Astronomy, University of British Columbia, Vancouver, BC V6T 1Z1, Canada; e-mail: krs@phas.ubc.ca

${ }^{1}$ Based on lectures given by MK at the Villa Olmo School on "The Dark Side of the Universe," 14-18 May 2007 and by KS at the XIX Heidelberg Physics Graduate Days, 8-12 October 2007. 
simple ("squiggly-line") arguments for the existence of dark matter in clusters and galaxies, as well as the arguments for why it is nonbaryonic. The motivation will be to provide insight into the evidence and arguments, rather than to summarize results from the latest state-of-the-art applications of the techniques.

Likewise, construction of particle-physics models for dark matter has become a huge industry, accelerated quite recently, in particular, with anomalous cosmic-ray and diffuse-background results [1,2]. Again, we will not attempt to survey these recent developments and focus instead primarily on the basic arguments for particle dark matter. In particular, there has developed in the theoretical literature over the past twenty years a "standard" weakly-interacting massive particle (WIMP) scenario, in which the dark-matter particle is a particle that arises in extensions (e.g., supersymmetry [3] or universal extra dimensions [4]) of the standard model that are thought by many particle theorists to provide the best prospects for new-physics discoveries at the Large Hadron Collider (LHC). We therefore describe this basic scenario. More detailed reviews of weakly-interacting massive particles, the main subject of this article, can be found in Refs. [3, 5, 6].

After describing the standard WIMP scenario, we provide a brief sampling of some ideas for "non-minimal" WIMPs, scenarios in which the WIMP is imbued with some additional properties, beyond simply those required to account for dark matter. We also briefly discuss some other attractive ideas (axions and sterile neutrinos) for WIMPs. Exercises are provided throughout.

\section{Astrophysical evidence}

It has been well established since the 1930s that there is much matter in the Universe that is not seen. It has also been long realized, and particularly since the early 1970s, that much of this matter must be nonbaryonic. The evidence for a significant quantity of dark matter accrued from galactic dynamics, the dynamics of galaxy clusters, and applications of the cosmic virial theorem. The evidence that much of this matter is nonbaryonic came from the discrepancy between the total matter density $\Omega_{m} \simeq 0.2-0.3$ (in units of the critical density $\rho_{c}=3 H_{0}^{2} / 8 \pi G$, where $H_{0}$ is the Hubble parameter), obtained from such measurements, and the baryon density $\Omega_{b} \simeq 0.05$ required for the concordance between the observed light-element $(\mathrm{H}, \mathrm{D}$, ${ }^{3} \mathrm{He},{ }^{4} \mathrm{He},{ }^{7} \mathrm{Li}$ ) abundances with those predicted by big-bang nucleosynthesis [7], the theory for the assembly of light elements in the first minutes after the big bang.

Rather than review the historical record, we discuss the most compelling arguments for nonbaryonic dark matter today as well as some observations most relevant to astrophysical phenomenology of dark matter today. 


\subsection{Galactic rotation curves}

The flatness of galactic rotation curves has provided evidence for dark matter since the 1970's. These measurements are particularly important now not only for establishing the existence of dark matter, but particularly for fixing the local dark-matter density, relevant for direct detection of dark matter. We live in a typical spiral galaxy, the Milky Way, at a distance $\sim 8.5 \mathrm{kpc}$ from its center. The visible stars and gas in the Milky Way extend out to a distance of about $10 \mathrm{kpc}$. From the rotation curve, the rotational velocity $v_{c}(r)$ of stars and gas as a function of Galactocentric radius $r$, we can infer the mass $M_{<}(r)$ of the Galaxy enclosed within a radius $r$. If the visible stars and gas provided all the mass in the Galaxy, one would expect that the rotation curve should decline at radii larger than the $10 \mathrm{kpc}$ extent of the stellar disk according to the Keplerian relation $v_{c}^{2}=G M_{o b s} / r$. Instead, one observes that $v_{c}(r)$ remains constant (a flat rotation curve) out to much larger radii, indicating that $M_{<}(r) \propto r$ for $r \gg 10 \mathrm{kpc}$ and thus that the Galaxy must contain far more matter than contributed by the stars and the gas.

Assuming a spherically symmetric distribution of matter, the mass inside a radius $r$ is given by

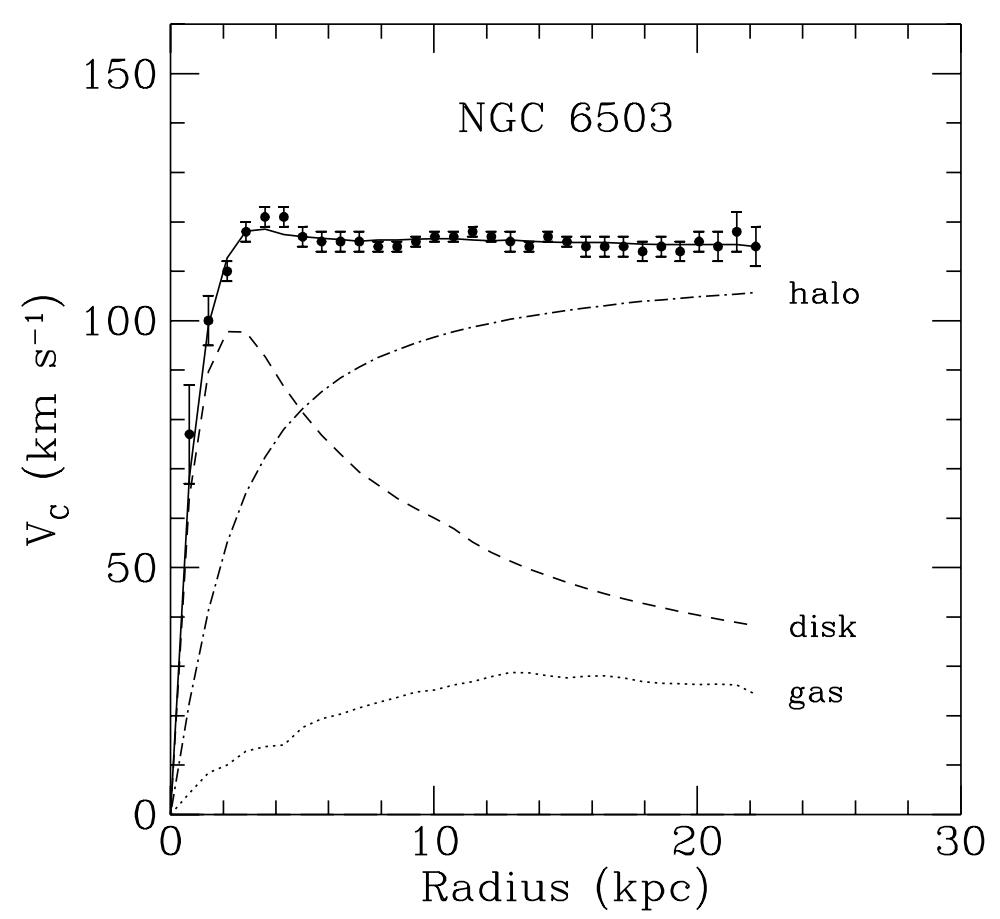

Fig. 1: Measured rotation curve of NGC6503 with best fit and contributions from halo, disk and gas. From Ref. [8] 


$$
M_{<}(r)=4 \pi \int_{0}^{r} \rho\left(r^{\prime}\right) r^{\prime 2} d r^{\prime}
$$

An estimate for the distribution of dark matter in the Galaxy can be obtained from the behavior of the rotation curve in the inner and outer galaxy. For example, the density distribution for the cored isothermal sphere, given by,

$$
\rho(r)=\rho_{0} \frac{R^{2}+a^{2}}{r^{2}+a^{2}},
$$

where $R \sim 8.5 \mathrm{kpc}$ is our distance from the Galactic center and $\rho_{0}$ is the local darkmatter density, provides a qualitatively consistent description of the data. For large $r, \rho \sim r^{-2} \Rightarrow M(r) \propto r \Rightarrow v \sim$ const, while for small $r, \rho \sim$ const $\Rightarrow M(r) \propto$ $r^{3} \Rightarrow v \propto r$. Eq. (2) describes a 2-parameter family of density profiles and by fitting the observed data one finds a scale radius $a \sim 3-5 \mathrm{kpc}$ and local matter density $\rho_{0} \sim 0.4 \mathrm{GeV} \mathrm{cm}^{-3}$; the uncertainties arise from standard error in the rotation-curve measurements and from uncertainties in the contribution of the stellar disk to the local rotation curve. Because the dark matter is moving in the same potential well, the velocity dispersion of the dark matter can be estimated to be $\left\langle v_{\mathrm{dm}}^{2}\right\rangle^{1 / 2} \sim 300 \mathrm{~km} / \mathrm{sec}$. The simplest assumption is that the dark matter has a Maxwell-Boltzmann distribution with $f(\vec{v}) \sim e^{-v^{2} / 2 \bar{v}^{2}}$, where $\bar{v} \sim 220 \mathrm{~km} / \mathrm{sec}$.

Exercise 1. Explain/estimate how $\rho_{0}$ would be affected if

- (a) the halo were flattened, keeping the rotation curve unaltered;

- (b) the profile were of the Navarro-Frenk-White (NFW) type: $\rho(r) \propto \rho_{c} /[r(r+$ $\left.r_{c}\right)^{2}$, keeping the local rotation speed the same;

- (c) the stellar contribution to the rotation curve was either increased or decreased.

\subsection{Galaxy Clusters}

Galaxy clusters are the largest gravitationally bound objects in the Universe. They were first observed as concentrations of thousands of individual galaxies, and early application of the virial theorem $v^{2} \sim G M / R$ (relating the observed velocity dispersion $v^{2}$ to the observed radius $R$ of the cluster) suggested that there is more matter in clusters than the stellar component can provide [9]. It was later observed that these galaxies are embedded in hot $\mathrm{x}$-ray-emitting gas, and we now know that clusters are the brightest objects in the $\mathrm{x}$-ray sky. The $\mathrm{x}$ rays are produced by hot gas excited to virial temperatures $T \sim \mathrm{keV}$ of the gravitational potential well of the dark matter, galaxies, and gas. A virial temperature $T \sim \mathrm{keV}$ corresponds to a typical velocity for the galaxies of $v \sim 10^{3} \mathrm{~km} / \mathrm{s}$.

Observations of clusters come from optical and x-ray telescopes and more recently via the Sunyaev-Zeldovich effect [10]. Several independent lines of evidence from clusters indicate that the total mass required to explain observations is much larger than can be inferred by the observed baryonic content of galaxies and gas. 


\subsubsection{Lensing}

Galaxy clusters exhibit the phenomenon of gravitational lensing $[11,12]$. Because the gravitational field of the cluster curves the space around it, light rays emitted from objects behind the cluster travel along curved rather than straight paths on their way to our telescopes [13]. If the lensing is strong enough, there are multiple paths from the same object, past the cluster, that arrive at our location in the Universe; this results in multiple images of the same object (e.g., a background galaxy or active galactic nucleus). Furthermore, because the light from different sides of the same galaxy travels along slightly different paths, the images of strongly lensed sources are distorted into arcs. For instance, HST observations of Abell 2218 show arcs and multiple images as shown in Fig. 2. If the lensing is weak, the images may become slightly elongated, even if they are not multiply imaged.

For a lensing cluster with total mass $M$ and impact parameter $d$ the deflection angle is of order

$$
\alpha \sim\left(\frac{G M}{d c^{2}}\right)^{1 / 2} .
$$

Thus, from measurements of the deflection angle and impact parameter (which can be inferred by knowing the redshift to the lensing cluster and source), one can infer that the total mass $M$ of a cluster is much larger than the observed baryonic mass $M_{b}$.

Exercise 2. Suppose a massive particle with velocity $v$ is incident, with impact parameter $b$, on a fixed deflector of mass $M$. Calculate the deflection angle (using classical physics) due to scattering of this particle via gravitational interaction with the deflector. Show that you recover $\alpha=\left(G M / d c^{2}\right)^{1 / 2}$ in the limit $v \rightarrow c$, the velocity

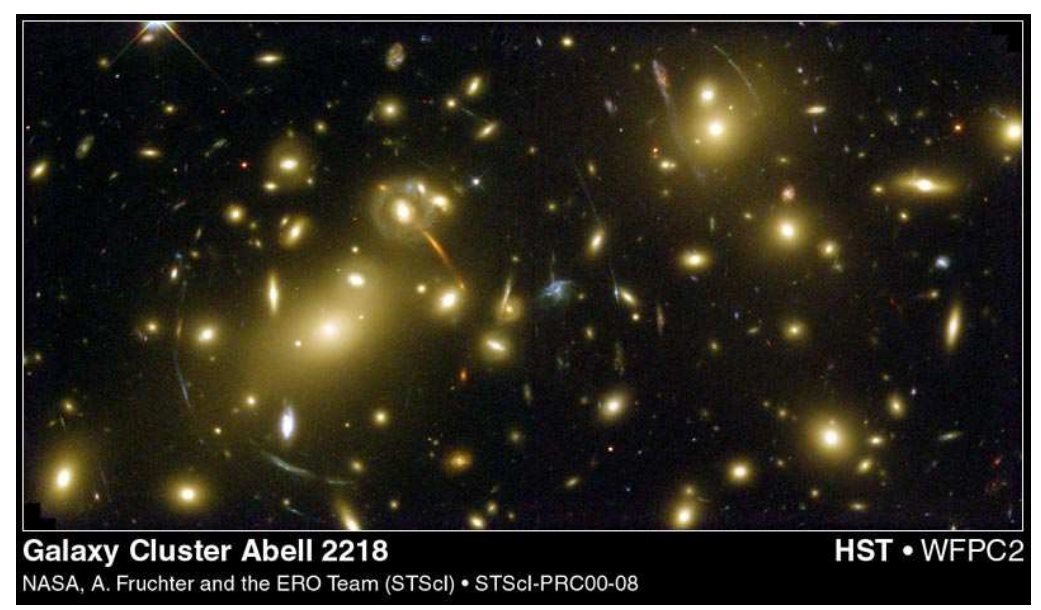

Fig. 2: Image of the galaxy cluster Abel 2218. Credits: NASA, Andrew Fruchter and the ERO team. 
at which light rays propagate. Actually, the correct general-relativistic calculation recovers this expression, but with an extra factor of 2 .

Exercise 3. Estimate the deflection angle $\alpha$ for lensing by a cluster of $M \sim 10{ }^{15} M_{\odot}$ and for an impact parameter of $1 \mathrm{Mpc}$.

\subsubsection{Hydrostatic equilibrium}

In a relaxed cluster, the temperature profile $T(r)$ of gas, as a function of radius $r$, can be inferred using the strength of the emission lines, and the electron number density $n_{e}(r)$ can be inferred using the the x-ray luminosity $L(r)$. Combined, these observations give an estimate of the radial pressure profile $p(r) \propto n_{e}(r) k_{B} T(r)$. In steady state, a gravitating gas will satisfy the equation of hydrostatic equilibrium,

$$
\frac{d p}{d r}=-G \frac{M_{<}(r) \rho_{\mathrm{gas}}(r)}{r^{2}} .
$$

Here, $M_{<}(r)$ is the total (dark matter and baryonic gas) mass enclosed by a radius $r$ and $\rho_{\text {gas }}(r)$ is the density at radius $r$. Eq. (4) can be used to determine the total mass $M$ of the cluster. Comparison with the observed baryonic mass $M_{b}$ again shows that $M \gg M_{b}$. In particular, observations using the x-ray satellites XMM-Newton and Chandra indicate that the ratio of baryonic matter to dark matter in clusters is $\Omega_{b} / \Omega_{m} \sim 1 / 6$. Additional constraints to the cluster-gas distribution can be obtained from the Sunyaev-Zeldovich (SZ) effect. This is the upscattering of cosmic microwave background (CMB) photons by hot electron gas in the cluster; the magnitude of the observed CMB-temperature change is then proportional to the integral of the electron pressure through the cluster (see, e.g., [10]).

Exercise 4. Estimate, in order of magnitude, the $x$-ray luminosity $L_{X}$ for a cluster with total mass $M \sim 10^{15} M_{\odot}$ and a baryon fraction 1/6 in hydrostatic equilibrium with maximum radius $R \sim \mathrm{Mpc}$.

Exercise 5. Assume the cluster in Exercise 4. is isothermal $(T(r)=T=$ const.) with a dark-matter distribution consistent with an NFW profile with $r_{c} \simeq R / 10$. Neglecting the self-gravity of the gas:

- (a) Show the properly normalized dark-matter density profile is approximately $\rho(r) \simeq(233 / 45) M_{c} /\left[r\left(r+r_{c}\right)^{2}\right]$, where $M_{c}=M_{<}\left(r_{c}\right)$ is the mass enclosed within the scale radius $r_{c}$. Determine $M_{<}(r)$ and $M_{c}$ and in terms of $M$ for this cluster.

- (b) Using your results from (a) solve Eq. 4 and show that the gas density profile in such an NFW cluster takes the form $\rho_{\text {gas }}(r) \propto\left(1+r / r_{c}\right)^{\Gamma r_{c} / r}$, where $\Gamma \propto$ $\left(G M_{c} \mu m_{p} / r_{c}\right) /\left(k_{B} T\right)$. 


\subsubsection{Dynamics}

According to the virial theorem, the velocity dispersion of galaxies is approximately $v^{2}(r) \sim G M_{<}(r) / r$, where $M_{<}(r)$ is the mass enclosed within a radius $r$. Therefore, from measurements of the velocity dispersion and size of a cluster (which can be determined if the redshift and angular size of the cluster are known), one can infer the total mass $M$. Once again, the total mass is much larger than the baryonic mass $M \gg M_{b}$.

Cluster measurements are by now well established, with many well-studied and very well-modeled clusters, and there is a good agreement of estimates of $M$ from dynamics, lensing, X-ray measurements, and the SZ effect. The current state of the art actually goes much further: one can now not only establish the existence of dark matter, but also map its detailed distribution within the cluster.

Exercise 6. Following Zwicky [14], use the virial theorem to find an approximate formula relating the average mass of a galaxy in a galaxy cluster to the observed size and velocity dispersion of the cluster assuming that the system is self-gravitating (and assuming only that the observed galaxies contribute to the mass of the system). What answer would Zwicky have found for the Coma cluster with modern data?

\subsection{Cosmic Microwave Background and Large-Scale Structure}

Measurements of the cosmic microwave background (CMB) radiation and largescale structure (LSS) of the Universe provide perhaps the most compelling evidence that the dark matter is non-baryonic and the most precise measurements of its abundance.

One obtains from $\mathrm{CMB}$ maps the angular power spectrum $C_{\ell}$ of $\mathrm{CMB}$ temperature anisotropies as a function of multipole $\ell$. If the temperature $T(\hat{\mathbf{n}})$ is measured as a function of position $\hat{\mathbf{n}}$ on the sky, then one can obtain the spherical-harmonic coefficients $a_{\ell m}=\int d \hat{\mathbf{n}} T(\hat{\mathbf{n}}) Y_{\ell m}^{*}(\hat{\mathbf{n}})$. The $C_{\ell}$ 's are then simply the variance of the spherical-harmonic coefficients: $C_{\ell}=\left\langle\left|a_{\ell m}\right|^{2}\right\rangle$. Theoretical predictions for the power spectrum depend on the values of cosmological parameters like the matter density $\Omega_{m} h^{2}$, the baryon density $\Omega_{b} h^{2}$, the cosmological constant $\Lambda$, the scalar spectral index $n_{s}$, the optical depth $\tau$ due to reionization, and the Hubble parameter $H_{0}$. One can thus determine these cosmological parameters by fitting precise measurements of the $C_{\ell}$ s to the theoretical predictions [16]. Current measurements provide detailed information on $C_{\ell}$ over the range $2<l<\mathscr{O}(1000)$, thus providing precise constraints to the cosmological parameters.

In the year 2000, data from the Boomerang and MAXIMA experiments (with supernova measurements) gave $\Omega_{m} h^{2}=0.13 \pm 0.05$ with error bars that shrink to \pm 0.01 taking into account other measurements or assumptions (e.g., LSS, Hubbleconstant, and supernova measurements, and/or the assumption of a flat Universe) [17]. Now, with WMAP, $\Omega_{m} h^{2}=0.133 \pm 0.006$ and $\Omega_{b} h^{2}=0.0227 \pm 0.0006$ [18]. 


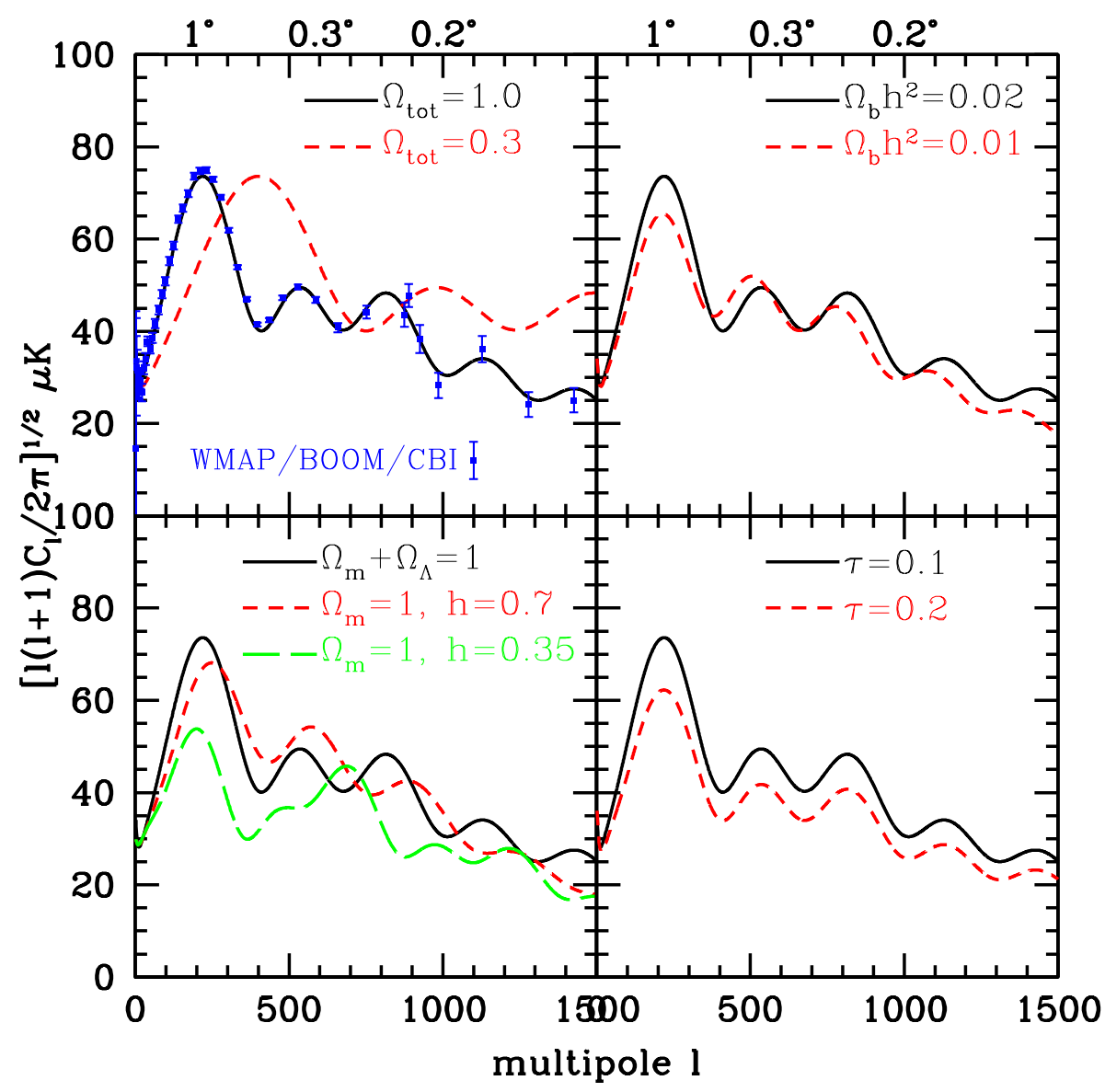

Fig. 3: Dependence of the CMB power spectrum on the cosmological parameters. From Ref. [15].

Exercise 7. Suppose that the temperature is measured with a Gaussian noise $\sigma_{T} \simeq$ $25 \mu \mathrm{K}$ in $N_{\text {pix }} \sim 10^{6}$ pixels on the sky. Estimate the rms temperature $\left\langle(\delta T / T)^{2}\right\rangle^{1 / 2}$ that results.

\section{Basic properties of dark matter}

Having established the existence of dark matter and presented the case that it is nonbaryonic, we now consider the requirements for a dark-matter candidate and discuss some possibilities. Every dark-matter candidate should satisfy several requirements: 
- Dark matter must be dark, in the sense that it must generically have no (or extremely weak) interactions with photons; otherwise it might contribute to the dimming of quasars, create absorption lines in the spectra of distant quasars [19], or emit photons. One way to quantify this is by assuming that dark-matter particles have a tiny charge $f e$ (where $e$ is the electron charge and $f \ll 1$ ), which can be quantitatively constrained [20].

- Self-interactions of the dark matter should be small. We can estimate the cross section for DM-DM scattering in the following way: if DM particles scatter less than once in the history of the Universe, then the mean free path is less than $\lambda=v_{D M} H_{0}^{-1} \sim\left(3 \times 10^{7} \mathrm{~cm} / \mathrm{sec}\right)\left(10^{17} \mathrm{sec}\right) \sim 3 \times 10^{24} \mathrm{~cm}$. Then, if the galactichalo density is $\rho_{D M} \sim 10^{-24} \mathrm{~g} / \mathrm{cm}^{3}$, the opacity for self-scattering in the galactic halo is $\kappa=\left(\rho_{D M} \lambda\right)^{-1}=\sigma / m \sim \mathrm{cm}^{2} / \mathrm{g}$. Thus, if the elastic-scattering cross section is $\sigma \gtrsim 10^{-24}(\mathrm{~m} / \mathrm{GeV}) \mathrm{cm}^{2}$, then $\kappa \gtrsim 1$ and the typical halo-dark-matter particle scatters more than once during the history of the Universe. If dark matter self-scattered, it would suffer gravothermal catastrophe: that is, in binary interactions of two dark-matter particles, one particle can get ejected from the halo, while the other moves to a lower-energy state at smaller radius. As this occurs repeatedly much of the halo evaporates and the remaining halo shrinks. Although a variety of arguments can constrain dark-matter self-interactions, stringent and very transparent constraints come from observations of the Bullet Cluster, the merger of two galaxy clusters, in which it is seen (from gravitational-lensing maps of the projected matter density) that the two dark-matter halos have passed through each other while the baryonic gas has shocked and is located between the two halos [21].

- Interactions with baryons must also be weak. Suppose baryons and dark matter interact. As an overdense region collapses to form a galaxy, baryons and dark matter would fall together, with photons radiated from this baryon-DM fluid. This would result in a baryon-DM disk, in contradiction with the more diffuse and extended dark-matter halos that are observed. If DM interacted with baryons other than gravitationally in the early Universe, the baryon-photon fluid would be effectively heavier (have a higher mass loading relative to radiation pressure) even before recombination, so that the baryon acoustic oscillations in the matter power spectrum and the CMB angular power spectrum would be modified [22].

- Dark matter cannot be made up of Standard Model (SM) particles, since most leptons and baryons are charged. The only potentially suitable SM candidate is the neutrino, but it cannot be dark matter because of the celebrated GunnTremaine bound [23], which imposes a lower bound on the masses of darkmatter particles that decoupled when relativistic. The argument is the following: The momentum distribution in the Galactic halo is roughly Maxwell-Boltzmann with a momentum uncertainty $\Delta p \sim m_{v}\langle v\rangle(\langle v\rangle \sim 300 \mathrm{~km} / \mathrm{sec})$, while the mean spacing between neutrinos is $\Delta x \sim n_{v}^{-1 / 3} \sim\left(\rho_{v} / m_{v}\right)^{-1 / 3}$. The Heisenberg uncertainty principle gives $\Delta x \Delta p \gtrsim \hbar$, which translates into a lower bound $m_{v} \gtrsim 50 \mathrm{eV}$. (This Heisenberg bound can actually be improved by a factor of 2 by using arguments involving conservation of phase space.) Stronger bounds $\left(m_{v} \gtrsim 300 \mathrm{eV}\right)$ can be obtained from dwarf galaxies which have higher phase-space densities. 


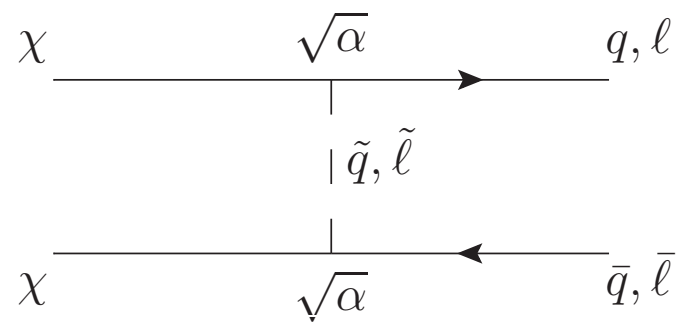

Fig. 4: An example of a Feynman diagram for annihilation of two WIMPs $\chi$ (neutralinos in this case) to fermion-antifermion pairs (where the fermions are either quarks $q$ or leptons $l$ ) via exchange of an intermediate-state squark $\tilde{q}$ or slepton $\tilde{l}$.

As discussed below, there will be a cosmological density of neutrinos left over from the big bang, with a density $\Omega_{v} h^{2} \sim 0.1\left(m_{v} / 10 \mathrm{eV}\right)$. The neutrinos of mass $m_{v} \gtrsim 300 \mathrm{eV}$ consistent with the Gunn-Tremaine bound would overclose the Universe. Thus, neutrinos are unable to account for the dark matter.

\section{Weakly Interacting Massive Particles (WIMPs)}

Perhaps the most attractive dark-matter candidates to have been considered are weakly-interacting massive particles (WIMPs). Many theories for new physics at the electroweak scale (e.g., supersymmetry, universal extra dimensions) introduce a new stable, weakly-interacting particle, with a mass of order $M_{\chi} \sim 100 \mathrm{GeV}$.

For example, in supersymmetric (SUSY) theories, the WIMP is the neutralino

$$
\tilde{\chi}=\xi_{\gamma} \tilde{\gamma}+\xi_{Z} \tilde{Z}^{0}+\xi_{h 1} \tilde{h}_{1}^{0}+\xi_{h 2} \tilde{h}_{2}^{0},
$$

a linear combination of the supersymmetric partners of the photon, $Z^{0}$ boson, and neutral Higgs bosons. Neutralinos are neutral spin-1/2 Majorana fermions. In theories with universal extra dimensions there are Kaluza-Klein (KK) states $\gamma_{K K}, Z_{K K}^{0}$, $H_{K K}^{0}$, which are neutral KK bosons. The candidates are stable (or quasi-stable; i.e., lifetimes greater than the age of the Universe $\tau \gg t_{U}$ ) and particle-theory models suggest masses $M_{\chi} \sim 10-10^{3} \mathrm{GeV}$.

In typical theories two WIMPs can annihilate to SM particles. For example, for a neutralino we have the tree-level diagram in Fig. 4, where $m_{\tilde{q}, \tilde{l}} \sim 100 \mathrm{GeV}$, so that $\sigma \sim \alpha^{2} m_{\tilde{q}, \tilde{l}^{-4}}^{-4} M_{\chi}^{2} \sim 10^{-8} \mathrm{GeV}^{-2}$. 


\subsection{WIMP Freezeout in Early Universe}

We now estimate the relic abundance of WIMPs in the standard scenario of thermal production (see, e.g., Ref. [24]). In the early Universe, at temperatures $T \gg M_{\chi}$, WIMPs are in thermal equilibrium and are nearly abundant as lighter particles, like photons, quarks, leptons, etc. Their equilibrium abundance is maintained via rapid interconversion of $\chi \chi$ pairs and particle-antiparticle pairs of Standard Model particles. When the temperature falls below the WIMP mass, however, the WIMP abundances become Boltzmann suppressed, and WIMPs can no longer find each other to annihilate. The remaining WIMPs constitute a primordial relic population that still exists today.

We now step through a rough calculation. To do so, we assume that the WIMP is a Majorana particle, its own antiparticle (as is the case for the neutralino, for example), although the calculation is easily generalized for WIMPs with antiparticles (e.g., KK WIMPs).

The annihilation rate for WIMPs is $\Gamma(\chi \chi \leftrightarrow q \bar{q}, \ell \bar{\ell}, \ldots)=n_{\chi}\langle\sigma v\rangle$, where $\sigma$ is the cross section for annihilation of two WIMPs to all lighter standard-model particles, $v$ is the relative velocity, and the angle brackets denote a thermal average. The expansion rate of the Universe is $H=(8 \pi G \rho / 3)^{1 / 2} \sim T^{2} / M_{\mathrm{Pl}}$ during the radiation era, where $\rho \propto T^{4}$. In the spirit of "squiggly lines" we have neglected factors like the effective number of relativistic degrees of freedom $g_{*}$ in the expansion rate, which the careful reader can restore for a more refined estimate.

By comparing these two rates, one can identify two different regimes:

- At early times, when $T \gg M_{\chi}, n_{\chi} \propto T^{3}$ and $\Gamma \gg H$ : particles scatter and annihilate many times during an Hubble time and this maintains chemical equilibrium.

- At late times, when $T \ll M_{\chi}, n_{\chi} \propto T^{3 / 2} e^{-M_{\chi} / T}$ (note that the chemical potential $\mu_{X}=0$ in the case of Majorana particles such as the neutralino) and $\Gamma \ll H$ : there can be no annihilations, and the WIMP abundance freezes out (the comoving number density becomes constant).

This sequence of events is illustrated in Fig. 5, which shows the comoving number density of WIMPs as a function of the inverse temperature in equilibrium (solid curve) and including freezeout (dashed curves).

Freezeout occurs roughly when $\Gamma\left(T_{f}\right) \sim H\left(T_{f}\right)$. For nonrelativistic particles, $n_{\chi}=g_{\chi}\left(M_{\chi} T / 2 \pi\right)^{3 / 2} e^{-M_{\chi} / T}$, so the freezeout condition becomes

$$
\left(M_{\chi} T_{f}\right)^{3 / 2} e^{-M_{\chi} / T_{f}} \sim \frac{T_{f}^{2}}{M_{P l}} \Rightarrow \frac{T_{f}}{M_{\chi}} \sim \ln \left[\frac{M_{P l} M_{\chi}^{3 / 2}\langle\sigma v\rangle}{T_{f}^{1 / 2}}\right]
$$

where the mass parameters are in GeV. Taking $\langle\sigma v\rangle \sim \alpha^{2} / M_{\chi}^{2}$, and taking as a first guess $T_{f} \sim M_{\chi}$, we finally find

$$
\frac{T_{f}}{M_{\chi}} \sim\left\{\ln \left[\frac{M_{P l} \alpha^{2}}{\left(M_{\chi} T_{f}\right)^{1 / 2}}\right]\right\}^{-1} \sim\left\{\ln \left[\frac{10^{19} 10^{-4}}{100}\right]\right\}^{-1} \sim \frac{1}{25}+\log \text { corrections, (7) }
$$


where the numerical values are characteristic electroweak-scale parameters (i.e. $\sigma \sim$ $10^{-8} \mathrm{GeV}^{-2}, M_{\chi} \sim 100 \mathrm{GeV}$ ).

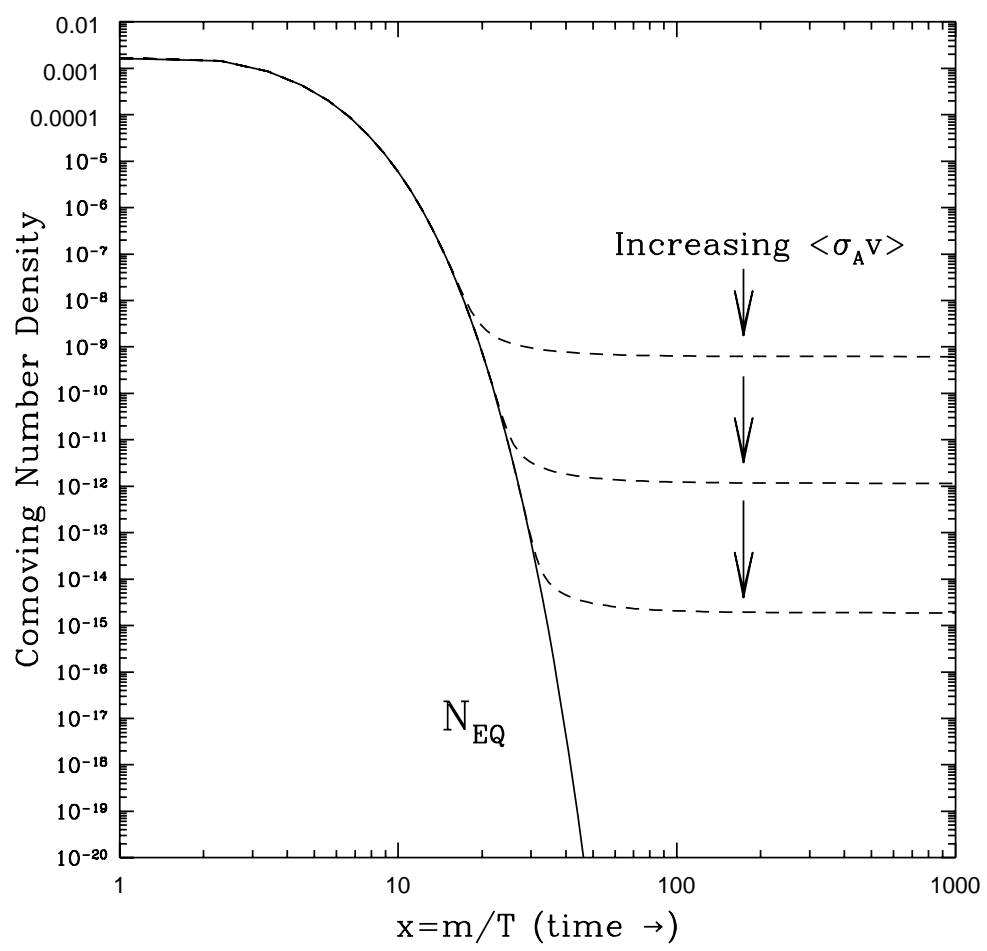

Fig. 5: Equilibrium (solid curve) and relic abundance (dashed curves) of WIMP particles. From Ref. [3].

At freezeout, the abundance relative to photons is

$$
\frac{n_{\chi}}{n_{\gamma}}=\frac{\Gamma\left(T_{f}\right) /\langle\sigma v\rangle}{T_{f}^{3}}=\frac{H\left(T_{f}\right) /\langle\sigma v\rangle}{T_{f}^{3}} \sim \frac{T_{f}^{2}}{M_{P l}\langle\sigma v\rangle T_{f}^{3}} \sim \frac{1}{M_{P l}\langle\sigma v\rangle T_{f}} \sim \frac{25}{M_{P l}\langle\sigma v\rangle M_{\chi}} .
$$

Today we know that

$$
\Omega_{\chi}=\frac{\rho_{\chi}}{\rho_{c}} \sim \frac{n_{\chi}^{0}}{n_{\gamma}^{0}} \frac{M_{\chi} n_{\gamma}^{0}}{\rho_{c}} \sim \frac{25}{M_{P l}\langle\sigma v\rangle} \frac{400 \mathrm{~cm}^{-3}}{10^{-6} \mathrm{GeV} \mathrm{cm}^{-3}}
$$

with no explicit dependence on the particle mass.

We thus obtain the observed abundance $\Omega_{\chi} h^{2} \sim 0.1$ for $\sigma \sim 10^{4}\left(0.1 \times 10^{19} \times\right.$ $\left.10^{-6}\right)^{-1} \mathrm{GeV}^{-2} \sim 10^{-8} \mathrm{GeV}^{-2}$ which turns out to be nearly exact, even though we 
have been a bit sloppy. A more precise calculation (including all the factors we have dropped) gives

$$
\Omega_{\chi} h^{2} \sim 0.1\left(\frac{3 \times 10^{-26} \mathrm{~cm}^{3} / \mathrm{sec}}{\langle\sigma v\rangle}\right)+\log \text { corrections }
$$

a remarkable result, as it implies that if there is a new stable particle at the electroweak scale, it is the dark matter.

As an aside, note that partial-wave unitarity of annihilation cross sections requires $\sigma \lesssim M_{\chi}^{-2}$, which means $\Omega_{\chi} h^{2} \gtrsim\left(M_{\chi} / 300 \mathrm{TeV}\right)^{2}$. This thus requires $\Omega_{\chi} h^{2} \lesssim$ $0.1, M_{\chi} \lesssim 100 \mathrm{TeV}$, without knowing anything about particle physics [25]. More precisely, this bound applies for point particles and does not apply if dark matter particles are bound states or solitons. If the interactions are strong, $\alpha \sim 1$, the bound is already saturated.

Although our arguments have been rough, one finds in SUSY and KK models that there are many combinations of reasonable values for the the SUSY or KK parameters that provide a WIMP with $\Omega_{\chi} h^{2} \sim 0.1$ for $10 \mathrm{GeV} \lesssim M_{\chi} \lesssim 1 \mathrm{TeV}$.

Exercise 8. Eq. (10) was derived assuming that the annihilation cross section $\langle\sigma v\rangle$ is temperature-independent. Redo the estimate for $\Omega_{\chi} h^{2}$ assuming that $\langle\sigma v\rangle \propto T^{n}$, where $n=1,2,3, \cdots$.

\subsection{Direct detection}

If WIMPs make up the halo of the Milky Way, then they have a local spatial density $n_{\chi} \sim 0.004\left(M_{\chi} / 100 \mathrm{GeV}\right)^{-1} \mathrm{~cm}^{-3}$ (roughly one per liter), and are moving with velocities $v \sim 200 \mathrm{~km} \mathrm{sec}^{-1}$. Moreover, there is a crossing symmetry between the annihilation $\chi \chi \rightarrow q \bar{q}$ and the elastic scattering $\chi q \rightarrow \chi q$ processes-apart from some kinematic factors the diagrams are more or less the same (as shown in Fig. 6) - so one expects roughly that the cross section $\sigma(\chi q \rightarrow \chi q) \sim \sigma(\chi \chi \rightarrow$ $q \bar{q}) \sim 10^{-36} \mathrm{~cm}^{2}$. One can therefore hope to detect a WIMP directly by observing its interaction with some target nucleus in a low-background detector composed, e.g., of germanium, xenon, silicon, sodium, iodine, or some other element.

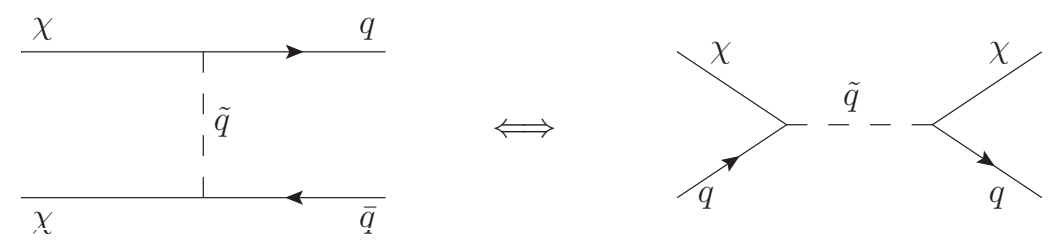

Fig. 6: Crossing symmetry between annihilation and scattering diagrams. 
At low energies, quarks are bound into nucleons and nucleons in turn are bound into nuclei, so the cross section one actually needs is $\sigma(\chi N \rightarrow \chi N)$ (where $N$ here stands for a nucleus). The calculation relating the $\chi q$ interaction to the $\chi N$ interaction requires both QCD and nuclear physics. It is complicated but straightforward. Here we will simply assume, for illustration, that $\sigma(\chi N \rightarrow \chi N) \sim \sigma(\chi q \rightarrow \chi q)$.

The rate at which a nucleus in the detector is hit by halo WIMPs is then

$$
R \sim n_{\chi} \sigma v \sim\left(0.004 \mathrm{~cm}^{-3}\right)\left(10^{-36} \mathrm{~cm}^{2}\right)\left(3 \times 10^{7} \frac{\mathrm{cm}}{\mathrm{sec}}\right) \sim 10^{-24} \mathrm{yr}^{-1}
$$

if there are $6 \times 10^{23} \mathrm{M} /(\mathrm{Ag})$ nuclei in a detector, for an atomic number $A \sim 100$ we expect to see $R \sim 10 / \mathrm{kg} / \mathrm{yr}$ events.

Let us estimate the recoil energy of a nucleus struck by a WIMP. If a WIMP of $M_{\chi} \sim 100 \mathrm{GeV}$ runs into a nucleus with $A \sim 100$, the momentum change is $\Delta p \sim M_{\chi} v$, and the nucleus recoils with an energy of order $E \sim(\Delta p)^{2} / 2 m \sim$ $\left(100 \mathrm{GeV} 10^{-3}\right)^{2}(100 \mathrm{GeV})^{-1} \sim 100 \mathrm{keV}$.

To do things more carefully, one has to account for the fact that the cross section one actually needs are the interaction cross sections with nuclei, and via the following steps,

$$
\sigma(\chi q) \underset{\mathrm{QCD}}{\longrightarrow} \sigma(\chi n), \sigma(\chi p) \underset{\text { nuclear physics }}{\longrightarrow} \sigma(\chi N),
$$

some theoretical uncertainties are introduced. One also finds that $\sigma(\chi N)$ is reduced relative to $\sigma(\chi q)$ by several orders of magnitude.

Qualitatively, there are two different types of interactions, axial and scalar (or spin-dependent and spin-independent). The first is described by the Lagrangian,

$$
\mathscr{L}_{\text {axial }} \propto \bar{\chi} \gamma^{\mu} \gamma_{5} \chi \bar{q} \gamma_{\mu} \gamma_{5} q
$$

which couples $\chi$ to the spin of unpaired nucleons; this works only for nuclei with spin, and the coupling is different for unpaired protons or neutrons. Through this interaction one expects $\sigma \propto \bar{s}^{2}$, where $\bar{s}$ is the average spin $\sim 1 / 2$ of the unpaired proton or neutron in nuclei with odd atomic number.

The second interaction is described by the Lagrangian,

$$
\mathscr{L}_{\text {scalar }} \propto \bar{\chi} \chi \bar{q} q,
$$

which couples $\chi$ to the mass of the nucleus, thus giving a cross section $\sigma \propto M^{2} \propto A^{2}$ (where $M$ and $A$ are the nuclear mass and atomic number), which implies higher cross sections for larger A. However, this scaling is only valid up to a limit. In fact, the momentum exchanged is $\Delta p \sim(100 \mathrm{GeV})\left(10^{-3}\right) \sim 0.1 \mathrm{GeV}$, and the nuclear radius is roughly $r \sim A^{1 / 3} 10^{-13} \mathrm{~cm}$, so from the uncertainty principle one has $r \Delta p \gtrsim$ 1 when

$$
\frac{(0.1 \mathrm{GeV})\left(10^{-13} \mathrm{~cm}\right)}{2 \times 10^{-14} \mathrm{GeV} \mathrm{cm}} A^{1 / 3} \gtrsim 1 \quad \Longrightarrow \quad A \gtrsim 10 .
$$


Detailed calculations show that the cross section for WIMP-nucleus elastic scattering does not increase much past $A \gtrsim 100$.

In experiments, people usually draw exclusion curves for the WIMP-nucleon cross section versus the WIMP mass $M_{\chi}$. The exclusion curves are less constraining both for low $M_{\chi}$ because of the low recoil energy, and for large $M_{\chi}$ because (for fixed local energy density $\rho_{\chi}$ ) the number density $n_{\chi} \propto M_{\chi}^{-1}$. To date, only the DAMA

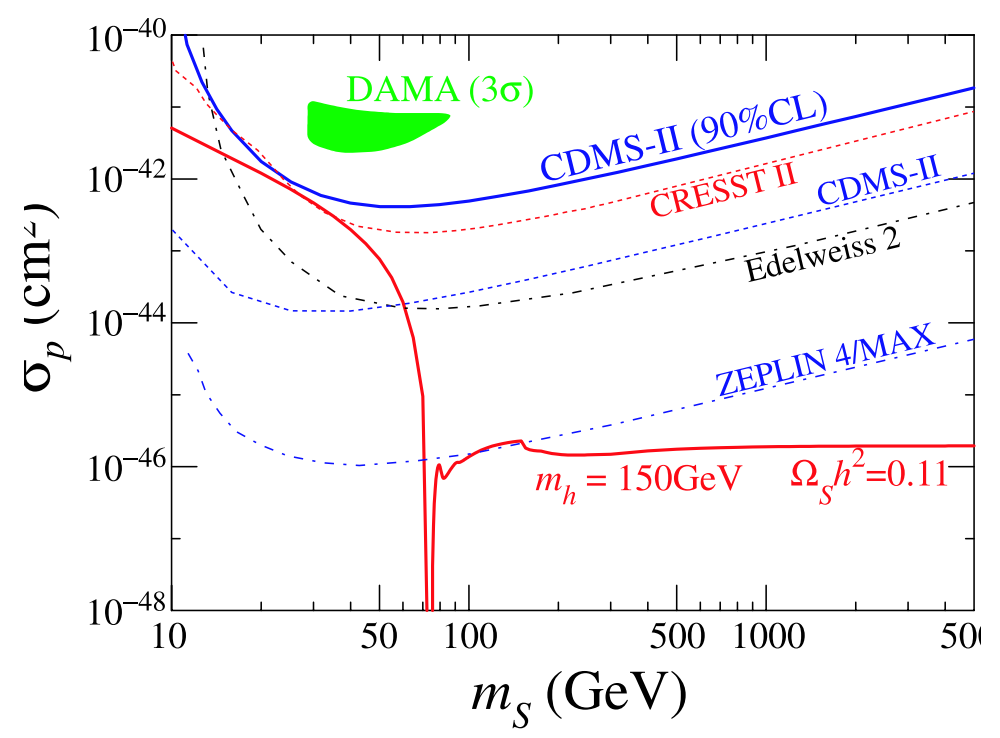

Fig. 7: Exclusion plot for the spin-independent dark-matter parameter space. The region favored by the DAMA annual modulation is inconsistent with the current bound (solid curve) from CDMS. The broken curves are forecasts for future experiments. We also show, for illustrative purposes only, predictions for a WIMP model with a lightest-Higgs-boson mass of $m_{h}=150 \mathrm{GeV}$.

experiment has reported a positive signal [26]. They used NaI, in which both nuclei have spin, one with an unpaired proton and the other with an unpaired neutron. The interpretation of their signal in terms of a WIMP with scalar interactions was ruled out by null results (at the time) from CDMS. An interpretation of their signal in terms of a spin-dependent WIMP-neutron interaction was ruled out by the null search in their Xe detector [27]. While the interpretation in terms of spin-dependent WIMP-proton scattering was consistent with null results from other direct searches [27], it was ruled out by null searches for energetic neutrinos from the Sun (see Fig. 8), as we explain below. The interpretation in terms of spin-dependent scattering is now also ruled out directly by null results from the COUPP experiment [28]. 


\subsection{Energetic v's from the Sun}

The escape velocity at the surface of the Sun is $v_{s} \sim 600 \mathrm{~km} / \mathrm{s}$, while at the center it is $v_{c} \sim 1300 \mathrm{~km} / \mathrm{s}$. If in passing through the Sun, a WIMP from the Galactic halo scatters from a nucleus (most likely a proton) therein to a velocity less than the escape velocity, then it is gravitationally trapped within the Sun. As the gravitationally-trapped WIMP passes through the Sun subsequently, it loses energy in additional nuclear scatters and thus settles to the center of the Sun. In this way, the number of WIMPs in the center of the Sun is enhanced. These WIMPs can then annihilate to standard model particles, through the same early-Universe processes that set their relic abundance [29]. Decays of the annihilation products (e.g., $W^{+} W^{-}, Z^{0} Z^{0}, \tau^{+} \tau^{-}, t \bar{t}, b \bar{b}, c \bar{c}, \ldots$ ) to neutrinos will produce energetic neutrinos that can escape from the center of the Sun. The neutrino energies are $E_{V} \sim[(1 / 3)-(1 / 2)] M_{\chi} \sim 100 \mathrm{GeV}$ and so cannot be confused with ordinary solar neutrinos, which have energies $\sim \mathrm{MeV}$. At night, these neutrinos will move up through the Earth. If the neutrino produces a muon through a charged-current interaction in the rock below a neutrino telescope (e.g., super-Kamiokande, AMANDA, or IceCube), the muon may be seen. In this way, one can search for these WIMPannihilation-induced neutrinos from the Sun.

\subsection{Cosmic rays from DM annihilation}

In the Galactic halo, one expects the annihilation processes $\chi \chi \rightarrow \cdots \rightarrow e^{+} e^{-}, p \bar{p}, \gamma \gamma$; detection of these products can be a signal of the presence of dark matter.

Exercise 9. Show that the annihilation process $\chi \chi \rightarrow e^{+} e^{-}$is suppressed for Majorana WIMPs as the relative velocity $v \rightarrow 0$.

\subsubsection{Positrons}

Because of Galactic magnetic fields, cosmic-ray positrons and antiprotons do not propagate in straight lines and will thus appear to us as a diffuse background. Continuum $e^{+}$'s from WIMP annihilation are difficult to separate from ordinary cosmicray positrons. It has been argued that indirect processes, such as the annihilation $\chi \chi \rightarrow W^{+} W^{-} \rightarrow e^{+} v e^{-} \bar{v}$ [30], will produce a distinctive bump in the positron spectrum at energies $E_{e} \lesssim M_{\chi}$ (direct annihilation of Majorana WIMPs to electronpositron pairs is suppressed at Galactic relative velocities), as illustrated in Fig. 9, and there has been tremendous excitement recently with the reported detection by the PAMELA experiment of such a bump [31]. However, it may be that nearby pulsars can also produce a bump in the positron spectrum [32], and more recent results from the Fermi Telescope [33] call the PAMELA result into questions. It will 


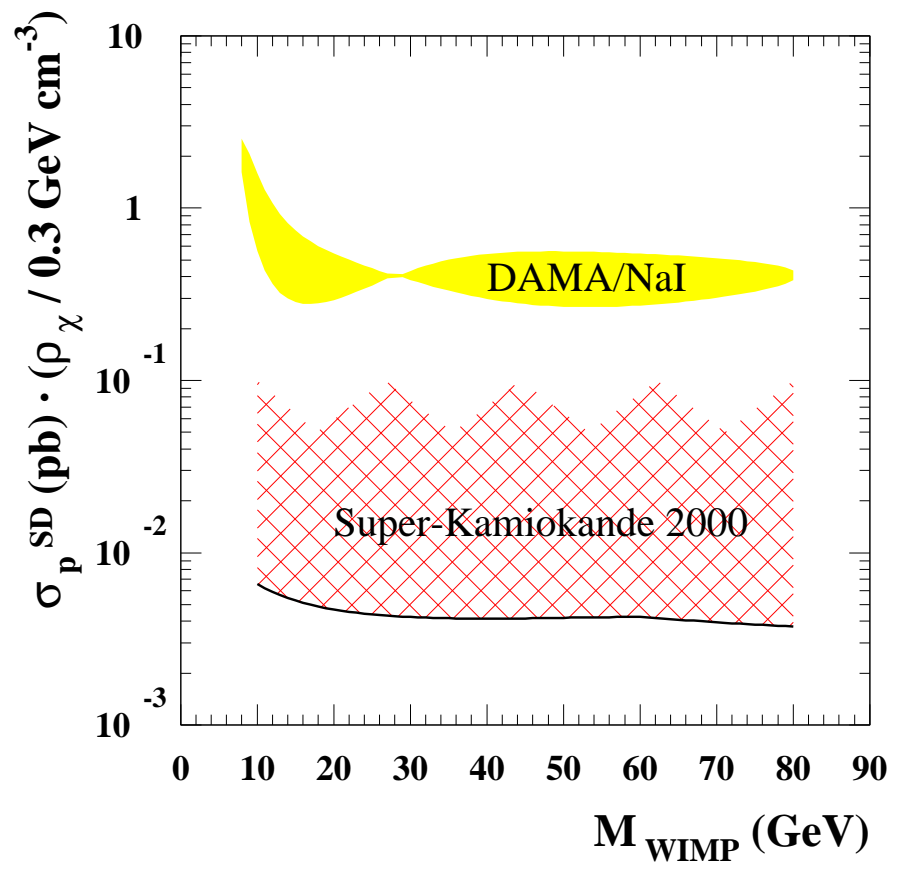

Fig. 8: The shaded region shows the parameter space (in WIMP mass versus SD WIMP-proton cross section) implied by the DAMA annual modulation for a WIMP with exclusively SD interactions with protons and no interaction with neutrons. The solid curve indicates the upper bound to the SD WIMP-proton cross section from null searches for neutrino-induced upward muons from the Sun; thus the cross hatched region is excluded [27].

thus be important to understand the possible pulsar signal, as well as the data, more carefully before the PAMELA excess can be attributed to WIMP annihilation.

\subsubsection{Antiprotons}

Likewise, it has also been argued that low-energy antiprotons from WIMP annihilation can be distinguished, through their energy spectrum, from the more prosaic cosmic-ray antiprotons produced by cosmic-ray spallation. Antiprotons can be produced by the decay of the standard WIMP-annihilation products, and the energy spectrum of such antiprotons is relatively flat at low energies. On the other hand, the energy spectrum of low-energy cosmic-ray antiprotons due to cosmic-ray spallation decreases at energies $E \lesssim \mathrm{GeV}$. This is because the process $\bar{p}+p_{I S M} \rightarrow p+p+\bar{p}+\bar{p}$ has an energy threshold, in the center of mass, of $E_{\mathrm{CM}}>4 m_{p}$. This requires the primary cosmic-ray momentum to be very high. Production of an antiproton with 
$E_{\bar{p}} \lesssim \mathrm{GeV}$ therefore requires that the antiproton be ejected with momentum opposite to that of the initial cosmic-ray proton, and the phase-space for this ejection is small.

\subsubsection{Gamma rays}

A final channel to observe WIMP annihilation is via gamma rays from WIMP annihilation. Direct annihilation of WIMPs to two photons, $\chi \chi \rightarrow \gamma \gamma$, through loop diagrams such as those shown in Fig. 10, produce monoenergetic photons, with energies equal to the WIMP mass. For $v \sim 10^{-3} c$, the photon energies would be $E_{\gamma}=E_{\chi}\left(1 \pm 10^{-3}\right)$, and one would see a narrow $\gamma$-ray line with $\Delta v / v \sim 10^{-3}$, superposed on a continuum spectrum produced by astrophysical processes; such a line would be difficult to mimic with traditional astrophysical sources. Decays of WIMP-annihilation products also produce a continuum spectrum of gamma rays at lower energies.

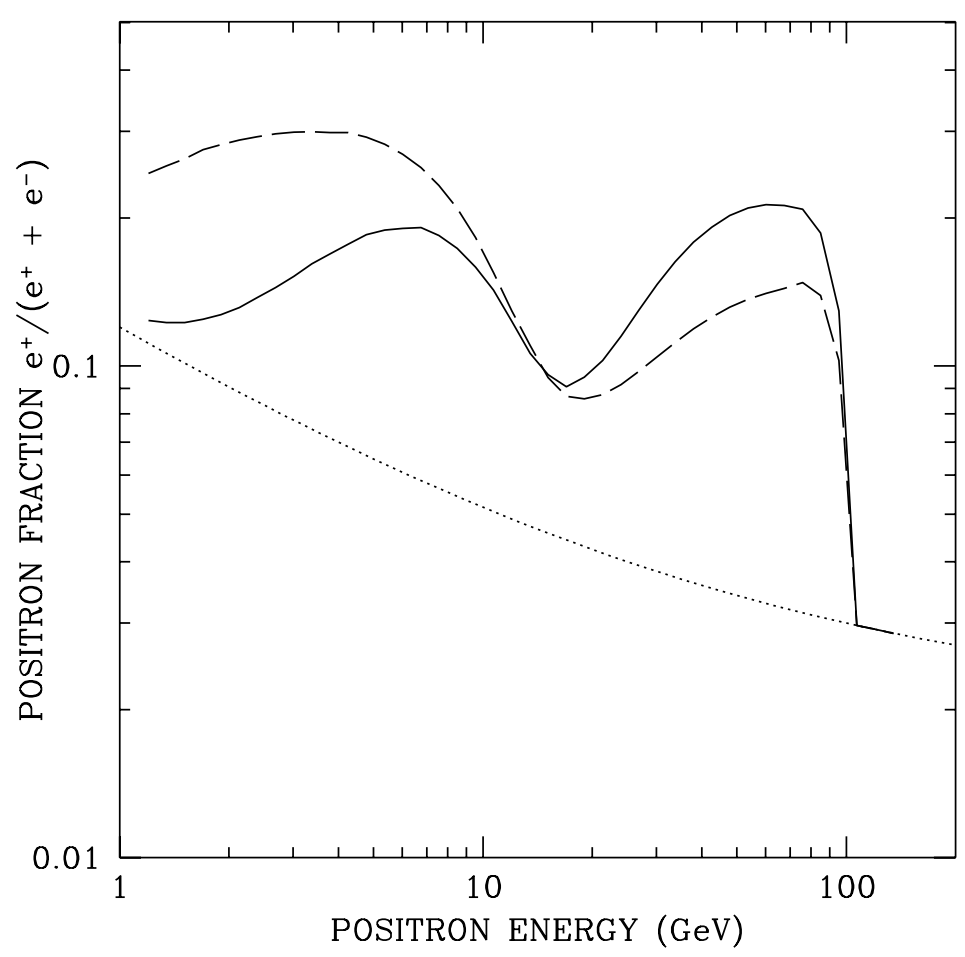

Fig. 9: The positron fraction, as a function of electron-positron energy, from annihilation of a $120 \mathrm{GeV}$ neutralino WIMP to gauge bosons. The different curves are for different cosmic-raypropagation models, and in both cases, the annihilation rate has been boosted by a factor of ten relative to the canonical (smooth-halo) value. From Ref. [30]. 


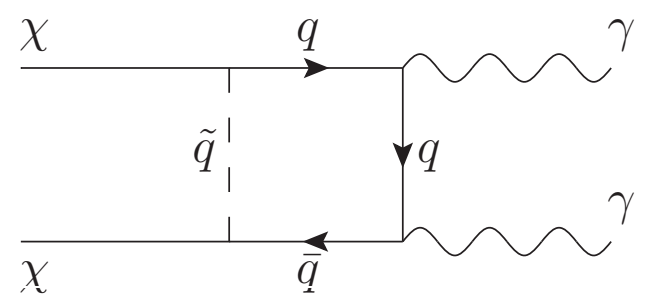

Fig. 10: Example of a Feynman diagram for annihilation of two neutralinos to two photons through a quark-squark loop.

The other advantage of gamma rays is that they propagate in straight lines. This opens up the possibility to distinguish gamma rays from WIMP annihilation from those from traditional sources through directionality - there should be a higher flux of WIMP-annihilation photons from places where WIMPs are abundant; e.g., the Galactic center. Another possibility is dwarf galaxies, which represent regions of high dark-matter density in the Milky Way halo. In general, the $\gamma$-ray flux (the number of photons per unit time-area-solid-angle) is given by

$$
\frac{d F}{d \Omega}=\frac{\left\langle\sigma_{\chi \chi \rightarrow \gamma \gamma} v\right\rangle}{4 \pi M_{\chi}^{2}} \int_{0}^{\infty} \rho^{2}(l) d l
$$

where the integral is taken along a given line of sight, $l$ is the distance along that line of sight, and $\rho(l)$ is the dark-matter density at that distance. (Note that if $\rho(r) \propto r^{-1}$ with Galactocentric radius $r$, as in an NFW profile, the intensity formally diverges, but the flux form any finite angular window around $r=0$ is finite.)

Exercise 10. Estimate the $\gamma$-ray flux from WIMP annihilation, for a given annihilation cross section (times relative velocity) $\langle\sigma v\rangle_{\text {ann }}$, in an angular window of radius $\sim 5$ degrees around the Galactic center. Estimate a characteristic $\langle\sigma v\rangle$ for WIMPs and evaluate your result for the gamma-ray flux for that value. How does it compare, in order of magnitude, with the sensitivity of the Fermi Gamma Ray Telescope?

\subsubsection{Galactic Substructure and Boost Factors}

The rate for annihilation, per unit volume, at any point in the Galactic halo is proportional to $\rho^{2}$, the square of the density at that point. The total annihilation rate in the halo, or in some finite volume of the halo, is then proportional to $\int d V \rho^{2}$, the integral, over that volume, of the density squared. In the canonical model, the halo density is presumed to vary smoothly with position in the Galaxy with some density profile; e.g., the isothermal profile in Eq. (2).

However, a Galactic halo forms as part of a recent stage in a sequence of hierarchical structure formation. In this scenario, small objects undergo gravitational 
collapse first; they then merge to form more massive objects, which then merge to form even more massive objects, etc. If some of these substructures remain partially intact as they merge into more massive halos, then any given halo (in particular, the Milky Way halo) may have a clumpy distribution of dark matter. This is in fact seen in simulations. What this implies is that the annihilation rate in the halo may be enhanced by a "boost factor" $B \propto\left\langle\rho^{2}\right\rangle /\langle\rho\rangle^{2}$, where the averages are over volume in the halo [34]. It may be possible to see angular variations in the gamma-ray signal from WIMP annihilation, due to this substructure [35, 36]. It has even been suggested that proper motions of nearby substructures may be visible [37], although Ref. [38] disputed this claim.

As we will see below, the first gravitationally-collapsed objects in WIMP models have masses in the range $10^{-6}-100$ Earth masses [39]. These objects may have densities several hundred times those of the mean halo density today. If so, and if these Earth-mass substructures survive intact through all subsequent generations of structure formation, then the boost factor $B$ may be as large as several hundred, implying much larger cosmic-ray fluxes than the canonical model predicts.

Such large boost factors are, however, unlikely. Simulations of recent generations in the structure-formation hierarchy show that while the tightly bound inner parts of halos may survive during merging, the outer parts are stripped. Ref. [40] developed an analytic model, parametrized in terms of a halo-survival fraction, to describe the (nearly) scale-invariant process of hierarchical clustering. This model then provided the boost factor $B$ in terms of that survival fraction. By comparing the results (cf., Fig. 11) of the analytic model for the local halo-density probability distribution function with subsequent measurements of the same distribution in simulations (Fig. 1 in Ref. [41]), one infers a small halo-survival fraction. The analytic model of Ref. [40] then suggests for this survival fraction no more than a small boost factor, $B \lesssim$ few.

\section{Variations and additions}

What we have described so far may be referred to as the minimal-WIMP scenario. In this scenario, the dark matter is a thermal relic with electroweak-scale cross sections. It is neutral and scatters from baryons with cross sections $\sim 10^{-40} \mathrm{~cm}^{2}$ (to within a few orders of magnitude). It has no astrophysical consequences in the postfreezeout Universe beyond its gravitational effects. However, the recent literature is filled with a large number of astrophysical anomalies for which explanations have been proposed in terms of non-minimal WIMPs, WIMPs endowed with extra interactions or properties. This is a vast literature, far too large to review here. We therefore provide here only a brief sampling, focusing primarily on those that we have worked on. 


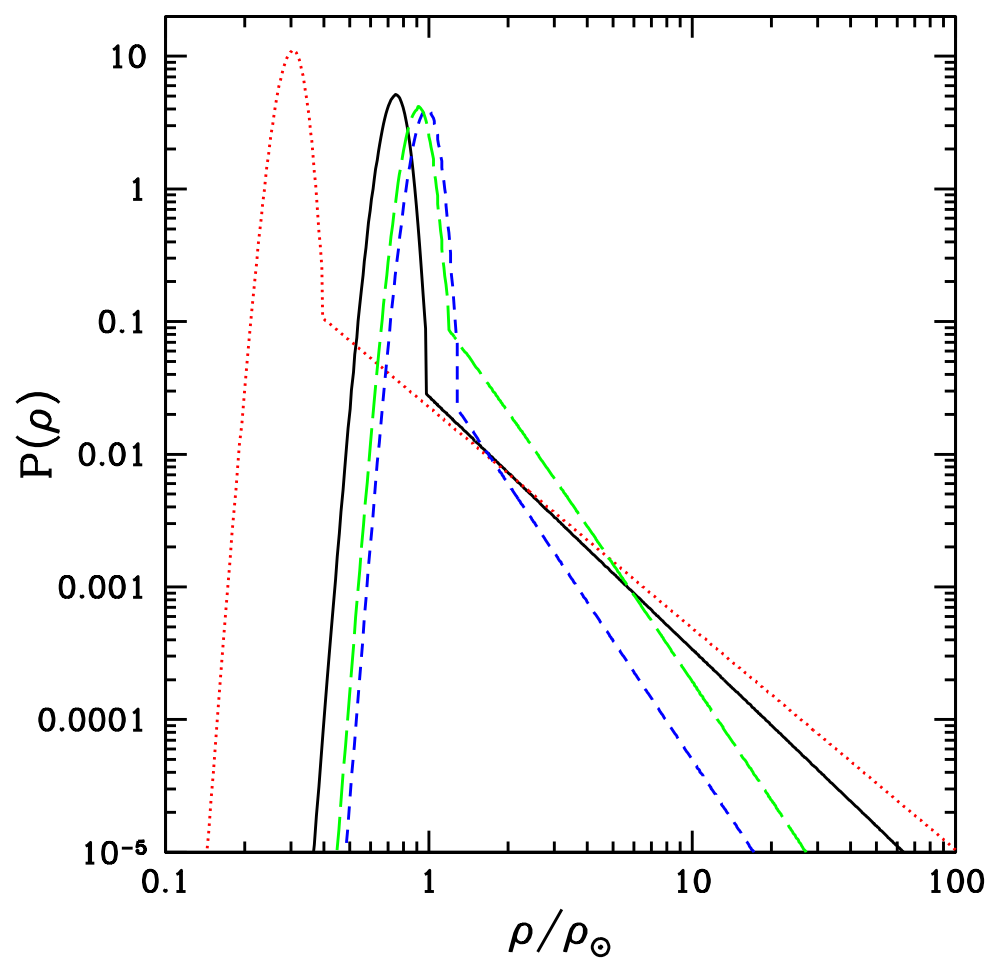

Fig. 11: The probability distribution function $P(\rho)$, due to substructure, for the local dark-matter density $\rho$, due to substructure, in units of the local halo density for a smooth halo. The different curves are for different substructure-survival fractions. The power-law tail is due to substructures. From Ref. [40].

\subsection{Enhanced relic abundance}

The calculation above of the freezeout abundance is the standard one in which it is assumed that the Universe is radiation-dominated at $T_{f} \sim 10-100 \mathrm{GeV}$. However, we have no empirical constraints to the expansion rate before big bang nucleosynthesis, which happens later, at $T_{B B N} \sim 1 \mathrm{MeV}$.

One can imagine other scenarios in which the WIMP abundance changes. For instance, suppose the pre-BBN Universe is filled with some exotic matter which has a stiff equation of state, $p_{s}=\rho_{s}$. This results in a scaling of the energy density of this stuff $\rho_{s} \propto a^{-6}$ with scale factor $a$ [42]. Such an equation of state may arise if the energy density is dominated by the kinetic energy of some scalar field. The equation of motion of a scalar field with a flat potential is

$$
\ddot{\varphi}+3 H \dot{\varphi}=0 \quad \Longrightarrow \quad \ln \dot{\varphi} \propto-3 \ln a
$$


which means that

$$
\rho=\frac{1}{2} \dot{\varphi}^{2} \propto a^{-6} .
$$

A stiff equation of state, or something that behaves effectively like it, may also arise, for example, in scalar-tensor theories of gravity or if there is anisotropic expansion in the early Universe.

Big-bang nucleosynthesis constrains the energy density of some new component of matter at a temperature $T \sim \mathrm{MeV}$ to be $\left(\rho_{6} / \rho_{\gamma}\right) \lesssim 0.1(T / \mathrm{MeV})^{2}$. Since $\rho_{s} / \rho_{\text {rad }} \propto T^{2}$, the expansion rate with this new stiff matter will at earlier times be $H(T) \lesssim H_{\text {st }}(T)(T / \mathrm{MeV})$, where $H_{\text {st }}(T)$ is the standard expansion rate. Neglecting the logarithmic dependence of the freezeout temperature $T_{f} \propto \ln \left[H \rho_{6} n_{\gamma}\right]$ on the expansion, the WIMP abundance with this new exotic matter will be

$$
\frac{n_{\chi}}{n_{\gamma}}=\frac{1}{n_{\gamma}} \frac{\Gamma}{\sigma v}=\frac{1}{n_{\gamma}} \frac{H}{\sigma v} \lesssim\left(\frac{n_{\chi}}{n_{\gamma}}\right)_{s t}\left(\frac{T}{\mathrm{MeV}}\right) \sim\left(\frac{n_{\chi}}{n_{\gamma}}\right)_{s t}\left(\frac{M_{\chi} / 25}{\mathrm{MeV}}\right) .
$$

Thus, for example, the relic abundance of an $M_{\chi} \sim 150 \mathrm{GeV}$ WIMP can be increased by as much as $\sim 10^{4}$ in this way $[42,43]$.

Exercise 11. Show that anisotropic expansion gives rise to a Friedmann equation that looks like that for a Universe with a new component of matter with $\rho \propto a^{-6}$. To do so, consider a Universe with metric $d s^{2}=d t^{2}-\left[a_{x}(t)\right]^{2} d x^{2}-\left[a_{y}(t)\right]^{2} d y^{2}-$ $\left[a_{z}(t)\right]^{2} d z^{2}$, with $a_{x}(t), a_{y}(t)$, and $a_{z}(t)$ different, and then derive the Friedmann equation for a Universe filled with homogeneous matter of density $\rho$.

\subsection{Kinetic decoupling}

There are two different kinds of equilibrium for WIMPs in the primordial bath. One is chemical equilibrium, which is maintained by the reactions

$$
\chi \chi \leftrightarrow f \bar{f} ;
$$

the other is kinetic equilibrium, maintained by the scattering

$$
\chi f \leftrightarrow \chi f
$$

The first reaction freezes out before the second, since $n_{f} \gg n_{\chi}$, where $f$ is any kind of light degree of freedom. However, $\sigma(v \chi \leftrightarrow v \chi) \propto E_{v}^{2}$ since the $v$ 's are Yukawa coupled, and $\sigma(\gamma \chi \leftrightarrow \gamma \chi) \propto E_{\gamma}^{2}$ since the photons are coupled by $\varepsilon_{\mu v \rho \sigma} k^{\mu} k^{v} \varepsilon^{\rho} \varepsilon^{\sigma}$ [44]. This means that $\Gamma(\chi f \leftrightarrow \chi f)$ drops rapidly and so kinetic freezeout happens not too much later than chemical freezeout.

Detailed calculations of the kinetic-decoupling temperature $T_{k d}$ show that $T_{k d}$ varies over 6 orders of magnitude in scans of the SUSY and UED parameter spaces [39]. During the time particles are chemically but not kinetically decou- 
pled, they have the same temperature of the thermal bath, which scales as $T_{\gamma} \propto a^{-1}$, and after that, $T_{\chi}=p_{\chi}^{2} / 2 M_{\chi} \propto a^{-2}$. So, density perturbations $\delta \rho_{\chi} / \rho_{\chi}$ are suppressed on $\lambda_{\text {phys }} \sim H^{-1}$ while the WIMPs are kinetically coupled. The cutoff in the power spectrum $P(k)$ is at physical wavenumber $k_{c}=H\left(T_{k d}\right)$, so if $T_{k d}$ decreases, also $k_{c}$ decreases. We expect power suppressed at mass scales $M<M_{c}$, where $M_{c} \sim 10^{-4}-10^{2} M_{\oplus}$ is the mass enclosed in the horizon at $T_{k d}$, as shown in Fig. 12 [39].

Exercise 12. Derive the mass $M_{k d}$ enclosed within the horizon at a temperature $T_{k d}$.

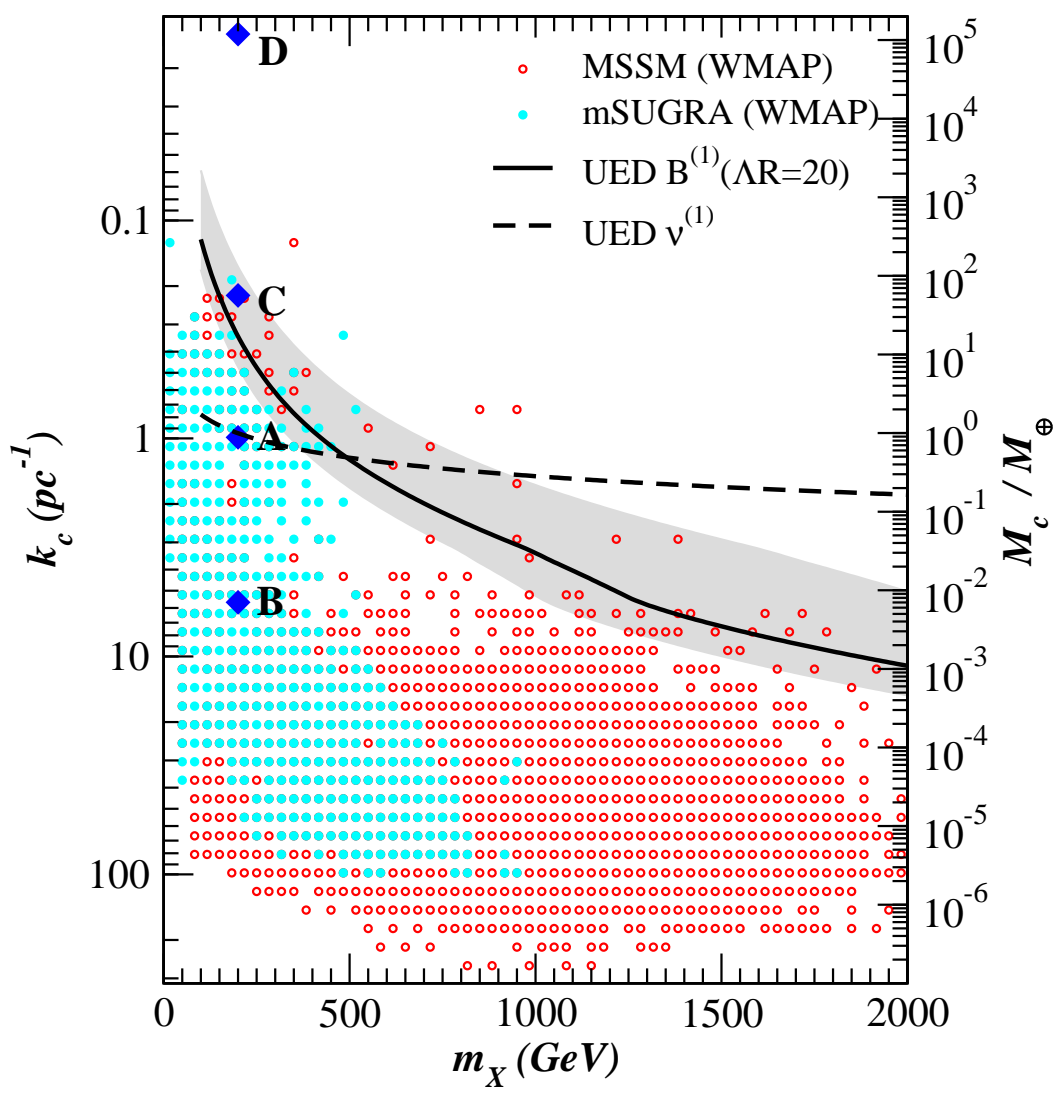

Fig. 12: The wavenumber and mass scale at which the primordial power spectrum is cut off due to kinetic decoupling of WIMPs in supersymmetric and UED models for WIMPs. From Ref. [39]. 


\subsection{Particle Decay and Suppression of Small Scale Power}

It might be the case that dark matter is produced by the decay of a metastable particle that was once in kinetic equilibrium with the thermal bath. For instance, although the dark matter cannot be a charged particle it might be produced by the decay of a charged particle. The growth of perturbation modes that enter the horizon prior to the decay of the charged particle will be suppressed relative to the standard case due to the coupling to the thermal bath: growth of charged-particle density perturbations is suppressed since charged particles cannot move through the baryon-photon fluid. If one has $\chi^{+} \rightarrow \chi^{0}+e^{+}$, with $\tau \sim 3.5 \mathrm{yr}\left(z \sim 10^{7}\right)$, then the matter power spectrum $P(k)$ is suppressed on $k \gtrsim \mathrm{Mpc}^{-1}$ [45], while for shorter lifetimes structure will be suppressed for larger $k$ (smaller length scales). Models exhibiting charged-particle decay can be found in the parameter space of standard or minimal extensions of canonical WIMP (e.g., supersymmetric) scenarios [46]. While limits on energy injection and the formation of exotic bound states in big bang nucleosynthesis (BBN) constrain the fraction of the Universe bound up in charged particles [47] the suppression of power due to particle decay in the Universe remains a potentially observable effect of metastable particles. It is possible the metastable particle might remain in kinetic equilibrium via another interaction, or even if the particle is out of kinetic equilibrium the energy released in the decay process may impart the dark-matter particle with a velocity high enough to erase small-scale structure via free streaming [48].

Future measurements of high-redshift cosmic 21-cm fluctuations may provide a direct probe of modifications to the small-scale dark-matter power spectrum and other aspects of fundamental physics (see, e.g., [46, 49]).

Exercise 13. Derive the comoving wavenumber $k$ that enters the horizon at the time a particle of lifetime $\tau$ decays.

\subsection{Dipole dark matter}

While dark matter cannot be a charged particle it may (via higher order interactions) be endowed with an electric or magnetic dipole moment interactions of the form $[22,19]$,

$$
\mathscr{L}_{\text {dipole }} \propto \bar{\chi}_{i} \sigma_{\mu v}\left(\mu_{i j}+\gamma_{5} \mathscr{D}_{i j}\right) \chi_{j} F^{\mu v},
$$

Here, diagonal interaction terms $(i=j)$ are the magnetic $(\mu)$ or electric $(\mathscr{D})$ dipole moments of a particle $\chi$, while off-diagonal terms $(i \neq j)$ are referred to as transition moments between the lightest WIMP state $i$ and another, slightly heavier, WIMP state $j$. Such a dipole coupling to photons alters the evolution of dark-matter density perturbations and CMB anisotropies [22], although the strongest constraints to dipole moments comes from precision tests of the Standard Model for WIMP 


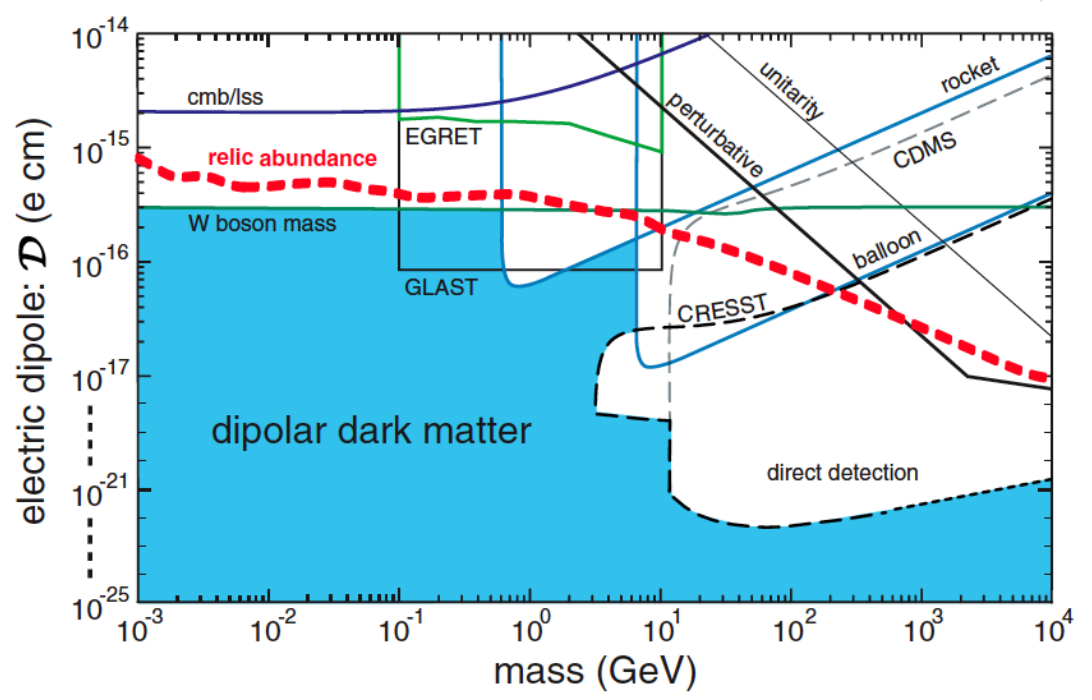

Fig. 13: Constraints to the dipole-mass parameter space for dark matter with an electric or magnetic dipole. From Ref. [22].

masses $M_{\chi} \lesssim 10 \mathrm{GeV}$ and direct-detection experiments for $M_{\chi} \gtrsim 10 \mathrm{GeV}[22,50]$; see Fig. 13 for the full constraints.

It may be possible to explain the results of the DAMA experiment using lowmass dipolar dark matter with a transition moment [50]. It may also be possible to look for the effects of a transition dipole moment in the absorption of high energy photons from distant sources [19].

Exercise 14. Calculate the cross section for elastic scattering of a particle with an electric dipole moment of magnitude $d$ from a nucleus with charge Ze.

\subsection{Gravitational constraints}

It is generally assumed that while dark matter may involve new physics, the gravitational interactions of the dark matter are standard. In other words, it is generally assumed that the gravitational force between two DM particles and between a darkmatter particle and a baryon is the same as that between two baryons. More precisely, the Newtonian gravitational force law between baryons that has been tested in the laboratory and in the Solar System reads $F_{b_{1} b_{2}}=G m_{1} m_{2} / d^{2}$. We then usually assume that the force between baryons and DM is $F_{b d}=G m_{b} m_{d} / d^{2}$, and also that the gravitational DM-DM force law is $F_{d_{1} d_{1}}=G_{d} m_{d_{1}} m_{d_{2}} / d^{2}$ with $G_{d}=G$. However, there is no empirical evidence that this is true at more than the order-unity level [51], and it has even been postulated that $G_{d}=2 G$ in order to account for the void 
abundance [52]. A similar behavior (an increase in the DM-DM force law) could also arise if there were a new long-range interaction mediated by a nearly massless scalar field $\varphi$ with Yukawa interactions $\varphi \bar{\psi} \psi$ with the DM field $\psi$. The difficulty in providing empirical constraints to this model is that measurements (e.g., gravitational lensing or stellar/galactic dynamics) of the dark-matter distribution determine only the gravitational potential $\Phi$ due to the dark-matter distribution, represented by some density $\rho_{d}(\vec{r})$, obtained through the Poisson equation $\nabla^{2} \Phi=4 \pi G \rho_{d}$. However, the same $\Phi$ can be obtained by replacing $\rho_{d} \rightarrow(1 / 2) \rho_{d}$ if we simultaneously replace $G \rightarrow 2 G$.

It turns out, though, that this exotic interaction can be constrained by looking at substructures in the Milky Way halo [53,54]. The Sagittarius dwarf galaxy, is dark-matter dominated, and it follows an elongated orbit around the Milky Way. When the dwarf reaches its point of closest approach to the Milky Way, the tidal forces it experiences in the Milky Way potential are largest. Stars are then stripped from the innermost and furthermost edge of the dwarf. Those from the innermost parts move at slightly larger velocities in the Galactic halo and at slightly smaller Galactocentric radii; they thus subsequently run ahead of the Sagittarius dwarf and form the leading tidal tail of the Sagittarius dwarf that is observed. Conversely, those stripped from the outer edge subsequently lag behind forming the trailing tidal tail that is observed. Observationally, the leading and trailing tails have roughly the same brightness, as expected. Suppose now that the DM-DM force law were modified to $G_{d}=f G$ with $f>1$. The dark-matter halo of the Sagittarius dwarf would then be accelerated toward the Milky Way center more strongly than the stellar component of the Sagittarius dwarf. The stellar component would then slosh to the furthermost edge. Then, when the dwarf reaches its point of closest approach to the Milky Way, stars are still stripped from the outer edge, forming a trailing tail. However, there are now no stars in the innermost edge to form the leading tail. The evacuation of stars from the leading tail is inconsistent with observations, and this leads, with detailed calculations, to a bound $G_{d}=G(1 \pm 0.1)$ to Newton's constant for DMDM interactions. In other words, dark matter and ordinary matter fall the same way, to within $10 \%$, in a gravitational potential well.

While Ref. [55] has more recently claimed to run a simulation of the tidal tails of the Sagittarius dwarf consistent with $G_{d}=2 G$, Ref. [56] has argued that the initial conditions for that simulation are self-inconsistent. Refs. [57, 58] argue that a new long-range DM-DM force law implies, under fairly general conditions, a weaker long-range DM-baryon force law, and they discuss and compare possible tests of such a scenario.

\subsection{Electromagnetic-like interactions for dark matter?}

Another possibility is that dark matter experiences long-range electromagnetic-like forces mediated by a dark massless photon that couples only to gravity. Of course, if the fine-structure constant $\alpha_{d}$ associated with this dark $U(1)$ symmetry is too large, 
then long-range dark forces will induce the dark matter to be effectively collisional. This constrains $\alpha_{d} \lesssim 0.005\left(M_{\chi} / \mathrm{TeV}\right)^{3 / 2}$ [59]. Far more restrictive constraints may arise from the development of plasma instabilities that may arise if there are (dark) positively and negatively charged dark-matter particles, but precise calculations of these effects remain to be done. See Refs. [59, 60] for more discussion of these models.

Exercise 15. Estimate the relic abundance of a dark-matter particle with dark charge $\alpha_{d}$ assuming that it annihilates to dark-photon pairs and assuming that the dark sector has the same temperature as the rest of the primordial plasma.

\section{Some other particle dark-matter candidates}

WIMP models are interesting for a number of reasons: (1) The correct relic density arises naturally if there is new physics at the electroweak scale; (2) there are good prospects for detection of these particles, if they are indeed the dark matter; and (3) there is synergy with the goals of accelerator searches (especially at the LHC) for new electroweak-scale physics.

Still, there are a large number of other particle candidates for dark matter. Here we discuss two, the sterile neutrino and the axion, which may also arise in extensions of the standard model and for which there are clear paths toward detection if they make up the dark matter.

\subsection{Sterile Neutrinos}

A convenient mechanism to introduce neutrino masses and explain their smallness by a minimal extension of the Standard Model is to add 3 right-handed neutrinos which are singlets under the SM gauge group. The mass matrix is taken to be of the form (for simplicity we consider only one family),

$$
\begin{aligned}
& v_{L} v_{R} \\
& v_{L}\left(\begin{array}{cc}
0 & M_{D} \\
v_{D} & M
\end{array}\right),
\end{aligned}
$$

where the $v_{L}$ and $v_{R}$ are left-handed and right-handed (weakly-interacting and sterile, respectively) fields.

In the see-saw mechanism, the Dirac mass is assumed to be tiny compared with the Majorana mass: i.e., $M_{D} \ll M$. The mass eigenstates then have masses $M_{1} \simeq$ $M_{D}^{2} / M \ll M$, and $M_{2} \simeq M$. For our purposes, it is advantageous to map the twodimensional $M_{D^{-}} M$ parameter space onto the $M_{s}-\theta$ parameter space, where $M_{s}$ is the mass of the sterile (heavier) neutrino and $\theta$ is the mixing angle between the two 
states. The active and sterile mass eigenstates can then be written

$$
\begin{aligned}
& \left|v_{a}\right\rangle=\cos \theta\left|v_{L}\right\rangle+\sin \theta\left|v_{R}\right\rangle, \\
& \left|v_{s}\right\rangle=-\sin \theta\left|v_{L}\right\rangle+\cos \theta\left|v_{R}\right\rangle,
\end{aligned}
$$

where $\theta=M_{D} / M$.

Sterile neutrinos can be produced in the early Universe and have both (1) a lifetime longer than the age of the Universe and (2) a cosmological density $\Omega_{s} \sim 0.2$ if the sterile-neutrino mass is in the $\sim \mathrm{keV}$ regime [61].

The main decay mode of the sterile neutrino is then $v_{S} \rightarrow v v \bar{v}$, through the exchange of a $Z^{0}$ boson, as shown in Fig. 14. The decay rate and lifetime are

$$
\Gamma=\frac{G_{F}^{2} M_{S}^{5}}{96 \pi^{3}} \theta^{2} \quad \Rightarrow \quad \tau_{S}=\frac{\hbar}{\Gamma} \sim 10^{20} \sec \left(\frac{M_{S}}{\mathrm{keV}}\right)^{5} \theta^{-2} .
$$

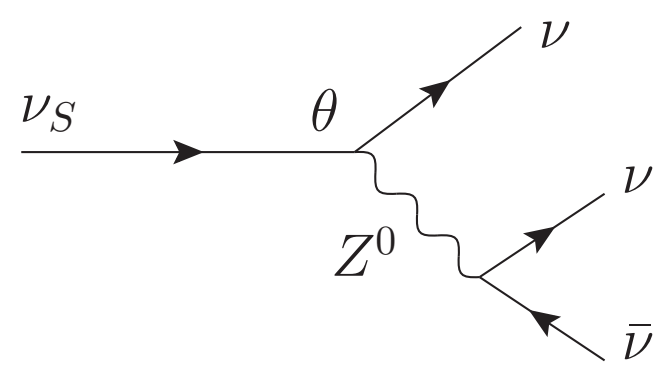

Fig. 14: Main decay channel for sterile neutrinos.

If the sterile neutrinos constitute the dark matter, then it must be that $\tau_{S} \gg$ $10^{17} \mathrm{sec}$, which is possible if $M_{S} \sim O(1) \mathrm{keV}$. This mass cannot however be too small, because of the Gunn-Tremaine limit from dwarf-spheroidal galaxies, which is $M_{S} \gtrsim 0.3 \mathrm{keV}$. A stronger constraint to the model comes from the X-ray emission in the radiative decay $v_{S} \rightarrow v \gamma$, through the diagram in Fig. 15. This produces an x-ray line that can be sought in the spectrum of, e.g., a galaxy cluster. While null searches for such lines (and from the diffuse cosmic x-ray background) provide $[62,63]$ stringent constraints to the model, there are still some regions in the $M_{s}-\theta$ parameter space that remain consistent with current constraints. This region may be probed, however, with future more sensitive $\mathrm{x}$-ray searches. One interesting extended application of sterile neutrino dark matter was its use as a potential mechanism for generating momentum-anisotropy during supernova to drive pulsar kicks [64]. See, for instance, Refs. [65, 66], for the current status of sterile neutrino dark matter. 


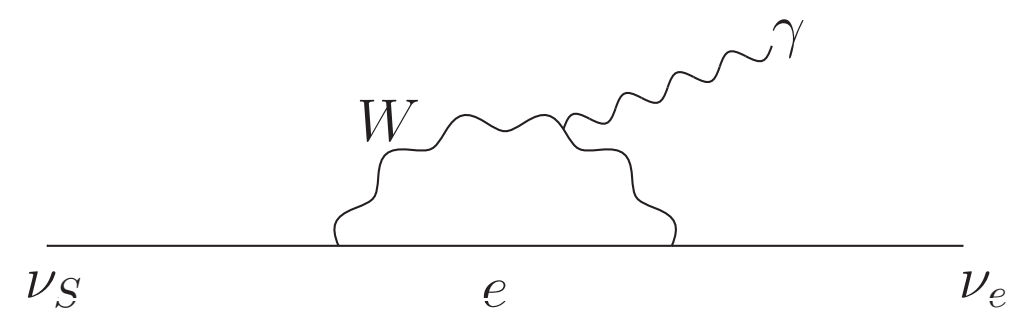

Fig. 15: Loop diagram for the decay $v_{s} \rightarrow v \gamma$.

\subsection{Axions}

Axions arise in the Peccei-Quinn (PQ) solution to the strong- $C P$ problem [67]. A global $U(1)_{P Q}$ symmetry is spontaneously broken at a scale $f_{a}$, and the CP-violating phase $\theta$ in the QCD Lagrangian becomes a dynamical field with a flat potential. At temperatures below the QCD phase transition, nonperturbative quantum effects break explicitly the symmetry and produce a non-flat potential that is minimized at $\theta \rightarrow 0$. The axion is the pseudo-Nambu-Goldstone boson of this near-global symmetry, the particle associated with excitations about the minimum at $\theta=0$. The axion mass is $m_{a} \simeq \mathrm{eV}\left(10^{7} \mathrm{GeV} / f_{a}\right)$, and its coupling to ordinary matter is $\propto f_{a}^{-1}$.

The Peccei-Quinn solution works equally well for any value of $f_{a}$. However, a variety of astrophysical observations and laboratory experiments constrain the axion mass to be $m_{a} \sim 10^{-4} \mathrm{eV}$. Smaller masses would lead to an unacceptably large cosmological abundance. Larger masses are ruled out by a combination of constraints from supernova 1987A, globular clusters, laboratory experiments, and a search for two-photon decays of relic axions.

Curiously enough, if the axion mass is in the relatively small viable range, the relic density is $\Omega_{a} \sim 1$, and so the axion may account for the halo dark matter. Such axions would be produced with zero momentum by a misalignment mechanism in the early Universe and therefore act as cold dark matter. During the process of galaxy formation, these axions would fall into the Galactic potential well and would therefore be present in our halo with a velocity dispersion near $270 \mathrm{~km} \mathrm{sec}^{-1}$.

It has been noted that quantum gravity is generically expected to violate global symmetries, and unless these Planck-scale effects can be suppressed by a huge factor, the Peccei-Quinn mechanism may be invalidated [68]. Of course, we have at this point no predictive theory of quantum gravity, and several mechanisms for forbidding these global-symmetry violating terms have been proposed [69]. Therefore, discovery of an axion might provide much needed clues to the nature of Planck-scale physics.

There is a very weak coupling of an axion to photons through the triangle anomaly, a coupling mediated by the exchange of virtual quarks and leptons. The axion can therefore decay to two photons, but the lifetime is $\tau_{a \rightarrow \gamma \gamma} \sim 10^{50} \mathrm{~s}\left(m_{a} / 10^{-5} \mathrm{eV}\right)^{-5}$ 
which is huge compared to the lifetime of the Universe and therefore unobservable. However, the $a \gamma \gamma$ term in the Lagrangian is $\mathscr{L}_{a \gamma \gamma} \propto a \vec{E} \cdot \vec{B}$ where $\vec{E}$ and $\vec{B}$ are the electric and magnetic field strengths. Therefore, if one immerses a resonant cavity in a strong magnetic field, Galactic axions that pass through the detector may be converted to fundamental excitations of the cavity, and these may be observable [70]. Such an experiment is currently underway [71] and has already begun to probe part of the cosmologically interesting parameter space (see the Figure in Ref. [72]), and it should cover most of the interesting region parameter space in the next few years.

Axions, or other light pseudoscalar particles, may show up astrophysically or experimentally in other ways. For example, the PVLAS Collaboration [73] reported the observation of an anomalously large rotation of the linear polarization of a laser when passed through a strong magnetic field. Such a rotation is expected in quantum electrodynamics, but the magnitude they reported was in excess of this expectation. One possible explanation is a coupling of the pseudoscalar $F \tilde{F}$ of electromagnetism to a low-mass axion-like pseudoscalar field. The region of the mass-coupling parameter space implied by this experiment violates limits for axions from astrophysical constraints, but there may be nonminimal models that can accommodate those constraints. Ref. [74] reviews the theoretical interpretation and shows how the interactions of axions and other axion-like particles may be tested with $\mathrm{x}$-ray re-appearance experiments. While the original PVLAS results have now been called into question Ref. [75], variations of the model may still be worth investigating.

\section{Conclusions}

Here we have reviewed briefly the basic astrophysical evidence for dark matter, some simple astrophysical constraints to its physical properties, and the canonical WIMP model for dark matter. We then discussed a number of variations of the canonical model, as well as some alternative particle dark-matter candidates. Still, we have only scratched the surface here, surveying only a small fraction of the possibilities for non-minimal dark matter. Readers who are interested in learning more are encouraged to browse the recent literature, where they will find an almost endless flow of interesting possibilities for dark matter, beyond those we have reviewed here.

\footnotetext{
Acknowledgements We thank Sabino Matarrese for initiating this collaboration during the Como summer school at which these lectures were given. We also thank the Aspen Center for Physics, where part of this review was completed. This work was supported at Caltech by DoE DEFG03-92-ER40701 and the Gordon and Betty Moore Foundation, and at the University of British Columbia by a NSERC of Canada Discovery Grant.
} 


\section{References}

1. D. P. Finkbeiner, Astrophys. J. 614, 186 (2004) [arXiv:astro-ph/0311547]; G. Dobler and D. P. Finkbeiner, Astrophys. J. 680, 1222 (2008) [arXiv:0712.1038 [astro-ph]]; D. Hooper, D. P. Finkbeiner, and G. Dobler, Phys. Rev. D 76, 083012 (2007) [arXiv:0705.3655 [astroph]]; P. Jean et al., Astron. Astrophys. 407, L55 (2003) [arXiv:astro-ph/0309484]; J. Knodlseder et al., Astron. Astrophys. 411, L457 (2003) [arXiv:astro-ph/0309442]; G. Weidenspointner et al., arXiv:astro-ph/0406178. A. W. Strong et al., Astron. Astrophys. 444, 495 (2005) [arXiv:astro-ph/0509290]; D. J. Thompson, D. L. Bertsch, and R. H. . O’Neal, arXiv:astro-ph/0412376.

2. O. Adriani et al. [PAMELA Collaboration], Nature 458, 607 (2009) [arXiv:0810.4995 [astro$\mathrm{ph}]]$.

3. G. Jungman, M. Kamionkowski and K. Griest, Phys. Rept. 267, 195 (1996) [arXiv:hep$\mathrm{ph} / 9506380]$.

4. H. C. Cheng, J. L. Feng, and K. T. Matchev, Phys. Rev. Lett. 89, 211301 (2002) [arXiv:hepph/0207125]; G. Servant and T. M. P. Tait, Nucl. Phys. B 650, 391 (2003) [arXiv:hepph/0206071]; for a review, see D. Hooper and S. Profumo, Phys. Rep. 453, 29 (2007) [arXiv:hep-ph/0701197].

5. L. Bergstrom, Rept. Prog. Phys. 63, 793 (2000) [arXiv:hep-ph/0002126].

6. G. Bertone, D. Hooper and J. Silk, Phys. Rept. 405, 279 (2005) [arXiv:hep-ph/0404175].

7. F. Iocco, G. Mangano, G. Miele, O. Pisanti and P. D. Serpico, Phys. Rept. 472, 1 (2009) [arXiv:0809.0631 [astro-ph]].

8. K. G. Begeman, A. H. Broeils and R. H. Sanders, Mon. Not. Roy. Astron. Soc. 249 (1991) 523.

9. F. Zwicky, Helv. Phys. Acta 6, 110 (1933).

10. R. A. Sunyaev and Y. B. Zeldovich, Ann. Rev. Astron. Astrophys. 18, 537 (1980).

11. A. Einstein, Science 84, 506 (1936).

12. F. Zwicky, Phys. Rev. 51, 290 (1937).

13. R. D. Blandford and R. Narayan, Ann. Rev. Astron. Astrophys. 30, 311 (1992).

14. F. Zwicky, Astrophys. J. 86, 217 (1937).

15. M. Kamionkowski, arXiv:0706.2986 [astro-ph].

16. G. Jungman, M. Kamionkowski, A. Kosowsky and D. N. Spergel, Phys. Rev. Lett. 76, 1007 (1996) [arXiv:astro-ph/9507080]; G. Jungman, M. Kamionkowski, A. Kosowsky and D. N. Spergel, Phys. Rev. D 54, 1332 (1996) [arXiv:astro-ph/9512139].

17. A. H. Jaffe et al. [Boomerang Collaboration], Phys. Rev. Lett. 86, 3475 (2001) [arXiv:astro$\mathrm{ph} / 0007333]$.

18. J. Dunkley et al. [WMAP Collaboration], Astrophys. J. Suppl. 180, 306 (2009) [arXiv:0803.0586 [astro-ph]].

19. S. Profumo and K. Sigurdson, Phys. Rev. D 75, 023521 (2007) [arXiv:astro-ph/0611129].

20. S. Davidson, S. Hannestad and G. Raffelt, JHEP 0005, 003 (2000) [arXiv:hep-ph/0001179].

21. S. W. Randall, M. Markevitch, D. Clowe, A. H. Gonzalez and M. Bradac, arXiv:0704.0261 [astro-ph].

22. K. Sigurdson, M. Doran, A. Kurylov, R. R. Caldwell and M. Kamionkowski, Phys. Rev. D 70, 083501 (2004) [Erratum-ibid. D 73, 089903 (2006)] [arXiv:astro-ph/0406355].

23. S. Tremaine and J. E. Gunn, Phys. Rev. Lett. 42 (1979) 407.

24. E. W. Kolb and M. S. Turner, "The Early universe," (Addison-Wesley, Redwood City, 1990).

25. K. Griest and M. Kamionkowski, Phys. Rev. Lett. 64, 615 (1990).

26. R. Bernabei et al. [DAMA Collaboration], Phys. Lett. B 480, 23 (2000).

27. P. Ullio, M. Kamionkowski and P. Vogel, JHEP 0107 (2001) 044 [arXiv:hep-ph/0010036].

28. E. Behnke et al. [COUPP Collaboration], Science 319, 933 (2008) [arXiv:0804.2886 [astro$\mathrm{ph}]]$.

29. J. Silk, K. A. Olive and M. Srednicki, Phys. Rev. Lett. 55, 257 (1985).

30. M. Kamionkowski and M. S. Turner, Phys. Rev. D 43 (1991) 1774. 
31. O. Adriani et al. [PAMELA Collaboration], arXiv:0810.4995 [astro-ph]; J. Chang et al. (ATIC) (2005), prepared for 29th International Cosmic Ray Conferences (ICRC 2005), Pune, India, 31 Aug 03 - 102005.

32. S. Profumo, arXiv:0812.4457 [astro-ph]; D. Hooper, P. Blasi and P. D. Serpico, JCAP 0901, 025 (2009) [arXiv:0810.1527 [astro-ph]].

33. A. A. Abdo et al. [The Fermi LAT Collaboration], Phys. Rev. Lett. 102, 181101 (2009) [arXiv:0905.0025 [astro-ph.HE]].

34. L. Bergstrom, J. Edsjo, P. Gondolo and P. Ullio, Phys. Rev. D 59, 043506 (1999) [arXiv:astroph/9806072]; L. Bergstrom, J. Edsjo and P. Ullio, Phys. Rev. D 58, 083507 (1998) [arXiv:astro-ph/9804050].

35. S. Ando and E. Komatsu, Phys. Rev. D 73, 023521 (2006) [arXiv:astro-ph/0512217]; S. Ando, E. Komatsu, T. Narumoto and T. Totani, Phys. Rev. D 75, 063519 (2007) [arXiv:astroph/0612467]; A. Cuoco, S. Hannestad, T. Haugbolle, G. Miele, P. D. Serpico and H. Tu, JCAP 0704, 013 (2007) [arXiv:astro-ph/0612559]; A. Cuoco, J. Brandbyge, S. Hannestad, T. Haugboelle and G. Miele, Phys. Rev. D 77, 123518 (2008) [arXiv:0710.4136 [astro-ph]]; J. M. Siegal-Gaskins, arXiv:0807.1328 [astro-ph].

36. S. K. Lee, S. Ando and M. Kamionkowski, arXiv:0810.1284 [astro-ph].

37. S. M. Koushiappas, Phys. Rev. Lett. 97, 191301 (2006) [arXiv:astro-ph/0606208];

38. S. Ando, M. Kamionkowski, S. K. Lee and S. M. Koushiappas, arXiv:0809.0886 [astro-ph].

39. S. Profumo, K. Sigurdson and M. Kamionkowski, Phys. Rev. Lett. 97 (2006) 031301 [arXiv:astro-ph/0603373].

40. M. Kamionkowski and S. M. Koushiappas, Phys. Rev. D 77, 103509 (2008) [arXiv:0801.3269 [astro-ph]].

41. M. Vogelsberger et al., arXiv:0812.0362 [astro-ph].

42. M. Kamionkowski and M. S. Turner, Phys. Rev. D 42 (1990) 3310.

43. S. Profumo and P. Ullio, JCAP 0311, 006 (2003) [arXiv:hep-ph/0309220].

44. X. 1. Chen, M. Kamionkowski and X. m. Zhang, Phys. Rev. D 64, 021302 (2001) [arXiv:astro$\mathrm{ph} / 0103452]$.

45. K. Sigurdson and M. Kamionkowski, Phys. Rev. Lett. 92, 171302 (2004) [arXiv:astro$\mathrm{ph} / 0311486]$.

46. S. Profumo, K. Sigurdson, P. Ullio and M. Kamionkowski, Phys. Rev. D 71, 023518 (2005) [arXiv:astro-ph/0410714].

47. M. Kaplinghat and A. Rajaraman, Phys. Rev. D 74, 103004 (2006) [arXiv:astro-ph/0606209]; M. Pospelov, Phys. Rev. Lett. 98, 231301 (2007) [arXiv:hep-ph/0605215]; K. Kohri and F. Takayama, Phys. Rev. D 76, 063507 (2007) [arXiv:hep-ph/0605243].

48. M. Kaplinghat, Phys. Rev. D 72, 063510 (2005) [arXiv:astro-ph/0507300].

49. A. Loeb and M. Zaldarriaga, Phys. Rev. Lett. 92, 211301 (2004) [arXiv:astro-ph/0312134]; M. Kleban, K. Sigurdson and I. Swanson, JCAP 0708, 009 (2007) [arXiv:hep-th/0703215].

50. E. Masso, S. Mohanty and S. Rao, arXiv:0906.1979 [hep-ph].

51. B. A. Gradwohl and J. A. Frieman, Astrophys. J. 398 (1992) 407.

52. S. S. Gubser and P. J. E. Peebles, Phys. Rev. D 70, 123510 (2004) [arXiv:hep-th/0402225].

53. M. Kesden and M. Kamionkowski, Phys. Rev. Lett. 97 (2006) 131303 [arXiv:astro$\mathrm{ph} / 0606566]$.

54. M. Kesden and M. Kamionkowski, Phys. Rev. D 74 (2006) 083007 [arXiv:astro-ph/0608095].

55. J. A. Keselman, A. Nusser, and P. J. E. Peebles, arXiv:0902.3452 [astro-ph.GA].

56. M. Kesden, arXiv:0903.4458 [astro-ph.CO].

57. S. M. Carroll, S. Mantry, M. J. Ramsey-Musolf and C. W. Stubbs, arXiv:0807.4363 [hep-ph].

58. S. M. Carroll, S. Mantry and M. J. Ramsey-Musolf, arXiv:0902.4461 [hep-ph].

59. L. Ackerman, M. R. Buckley, S. M. Carroll and M. Kamionkowski, Phys. Rev. D 79, 023519 (2009) [arXiv:0810.5126 [hep-ph]].

60. J. L. Feng, M. Kaplinghat, H. Tu and H. B. Yu, arXiv:0905.3039 [hep-ph].

61. S. Dodelson and L. M. Widrow, Phys. Rev. Lett. 72, 17 (1994) [arXiv:hep-ph/9303287].

62. A. Boyarsky, A. Neronov, O. Ruchayskiy, M. Shaposhnikov and I. Tkachev, Phys. Rev. Lett. 97 (2006) 261302 [arXiv:astro-ph/0603660]. 
63. A. Boyarsky, J. Nevalainen and O. Ruchayskiy, Astron. Astrophys. 471 (2007) 51 [arXiv:astro-ph/0610961].

64. A. Kusenko, Int. J. Mod. Phys. D 13 (2004) 2065 [arXiv:astro-ph/0409521].

65. M. Shaposhnikov, arXiv:astro-ph/0703673.

66. A. Kusenko, arXiv:0906.2968 [hep-ph].

67. R. D. Peccei and H. R. Quinn, Phys. Rev. Lett. 38, 1440 (1977); F. Wilczek, Phys. Rev. Lett. 40, 279 (1978); S. Weinberg, Phys. Rev. Lett. 40, 223 (1978).

68. M. Kamionkowski and J. March-Russell, Phys. Lett. B 282, 137 (1992) [arXiv:hepth/9202003]; R. Holman, S. D. H. Hsu, T. W. Kephart, E. W. Kolb, R. Watkins and L. M. Widrow, Phys. Lett. B 282, 132 (1992) [arXiv:hep-ph/9203206]; S. M. Barr and D. Seckel, Phys. Rev. D 46, 539 (1992).

69. N. Turok, Phys. Rev. Lett. 76, 1015 (1996) [arXiv:hep-ph/9511238]; R. Kallosh, A. D. Linde, D. A. Linde and L. Susskind, Phys. Rev. D 52, 912 (1995) [arXiv:hep-th/9502069]; E. A. Dudas, Phys. Lett. B 325, 124 (1994) [arXiv:hep-ph/9310260]; K. S. Babu and S. M. Barr, Phys. Lett. B 300, 367 (1993) [arXiv:hep-ph/9212219].

70. P. Sikivie, Phys. Rev. Lett. 51, 1415 (1983) [Erratum-ibid. 52, 695 (1984)].

71. S. Asztalos et al., Phys. Rev. D 64, 092003 (2001). S. J. Asztalos et al., Astrophys. J. 571, L27 (2002) [arXiv:astro-ph/0104200].

72. S. Eidelman et al. [Particle Data Group], Phys. Lett. B 592, 1 (2004). See, in particular, p. 394-397 of the review.

73. E. Zavattini et al. [PVLAS Collaboration], Phys. Rev. Lett. 96, 110406 (2006) [arXiv:hepex/0507107].

74. R. Rabadan, A. Ringwald and K. Sigurdson, Phys. Rev. Lett. 96, 110407 (2006) [arXiv:hep$\mathrm{ph} / 0511103]$.

75. A. S. .. Chou et al. [GammeV (T-969) Collaboration], Phys. Rev. Lett. 100, 080402 (2008) [arXiv:0710.3783 [hep-ex]]. 